\title{
CHIARITÉ
}

UNIVERSITÄTSMEDIZIN BERLIN

\section{Superviscous properties of the in vivo brain at large scales}

Helge Herthum, Sergio C. H. Dempsey, Abbas Samani, Felix Schrank, Mehrgan Shahryari, Carsten Warmuth, Heiko Tzschätzsch, Jürgen Braun, Ingolf Sack

\section{Document type}

Preprint (submitted version)

This version is available at

https://doi.org/10.17169/refubium-32093

\section{Citation details}

Herthum H, Dempsey SCH, Samani A, Schrank F, Shahryari M, Warmuth C, et al. Superviscous properties of the in vivo brain at large scales. [Online] Acta Biomaterialia. Elsevier BV; 2021. p. 393-404. DOI: 10.1016/j.actbio.2020.12.027

\section{Terms of use}

This work is licensed under a Creative Commons Attribution 4.0 International license: https://creativecommons.org/licenses/by/4.0/ 


\section{Superviscous properties of the in vivo brain at large scales.}

Helge Herthum ${ }^{1}$, Sergio C H Dempsey², Abbas Samani2,3,4, Felix Schrank ${ }^{1}$, Mehrgan Shahryari ${ }^{1}$, Carsten Warmuth ${ }^{1}$, Heiko Tzschätzsch ${ }^{1}$, Jürgen Braun ${ }^{5}$, Ingolf Sack ${ }^{1, *}$

${ }^{1}$ Department of Radiology, Charité - Universitätsmedizin Berlin, Berlin, Germany ${ }^{2}$ School of Biomedical Engineering, Western University, London, Ontario, Canada ${ }^{3}$ Department of Medical Biophysics, Western University, London, Ontario, Canada ${ }^{4}$ Department of Electrical and Computer Engineering, Western University, London, Ontario, Canada ${ }^{5}$ Institute of Medical Informatics, Charité - Universitätsmedizin Berlin, Berlin, Germany ${ }^{*}$ Corresponding author:

Ingolf Sack, $\mathrm{PhD}$

Department of Radiology

Charité - Universitätsmedizin Berlin

Charitéplatz 1

10117 Berlin, Germany

Tel +49 30450539058

Ingolf.sack@charite.de 


\begin{abstract}
There is growing awareness that brain mechanical properties are important for neural development and health. Yet, published values of brain stiffness differ by orders of magnitude from ex vivo to in vivo, pointing to a general lack of understanding of the complex mechanical behavior of brain tissue. We here show that there is no fundamental disparity between ex vivo and in vivo data when considering large-scale properties of the entire brain. Using numerical simulations and novel real-time magnetic resonance elastography we investigated the viscoelastic dispersion of the human brain in, so far, unexplored dynamic ranges from intrinsic brain pulsations at $1 \mathrm{~Hz}$ to externally induced harmonic vibrations at $40 \mathrm{~Hz}$. Surprisingly, we observed variations in brain stiffness over more than two orders of magnitude, suggesting that the in vivo human brain is superviscous on large scales with very low shear modulus of $42 \pm 13 \mathrm{~Pa}$ and relatively high viscosity of $6.6 \pm 0.3 \mathrm{~Pa} \cdot \mathrm{s}$ according to the two-parameter solid model. Our data shed light on the crucial role of fluid compartments including blood vessels and cerebrospinal fluid (CSF) for whole brain properties and provide, for the first time, an explanation for the variability of the mechanical brain responses to manual palpation, local indentation, and high-dynamic tissue stimulation as used in elastography.
\end{abstract}

Keywords: multifrequency MRE; intrinsic brain activation; low-frequency time-harmonic tissue stimulation; viscoelasticity; stiffness dispersion 


\section{Introduction}

The in-vivo mechanical properties of the brain are increasingly recognized as being tightly linked to neuronal development $\underline{1}$ and aging $\underline{2} \cdot \underline{3}$, myelination $\underline{4}$, functional activation $\underline{5}, \underline{6}$, memory performance $\underline{z}, \underline{\theta}$, body-mass index $\underline{9}$, and cerebrovascular perfusion $\underline{\underline{10}} \underline{\underline{11}}$ as well as pathophysiological processes including brain tumor progression $\underline{12-16}$, neuroinflammation17-19, and neuronal dementia20-23.

Elasticity, stiffness, and rigidity are synonymously used to refer to the major output parameter of clinical elastography, which is directly linked to the lengths of shear waves 24 . Shear oscillation rheometry or indentation techniques have been used as ground-truth in brain tissue for decades 25 . Interestingly, stiffness values can vary by orders of magnitude across testing modes (e.g., stretching vs. compression vs. shear $\underline{6} \underline{27}$ ), models (e.g., linear vs. nonlinear, isotropic vs anisotropic28-31), dynamic ranges (e.g., static vs. highdynamic32.33), tissue regions (e.g., full brain or white matter vs. cortical tissue or deepgray matter $\underline{34}, \underline{35})$, specimens (e.g., human vs. mouse brain $\underline{36}, \underline{37})$, or viability status (e.g., in vivo vs. in situ, post mortem, or ex vivo $\underline{38}, \underline{39}$ ) as reported before 25,40 .

It is a peculiarity of mechanical testing of soft biological tissues, in particular the brain, that there is an obvious discrepancy between local mechanical tests utilizing quasi-static deformations (on the order of 400 to 600 Pa1ㅡ), in vivo properties measured by magnetic resonance elastography (MRE) (1000 to $3500 \mathrm{~Pa}$ for the human brain $\underline{7}$, 10,000 to 20,000 $\mathrm{Pa}$ for the mouse brain $\underline{36}$ ), and reports of fresh ex vivo brains that have a very low flexural modulus 38 confirmed by our whole brain stiffness measurement that led to a value of 100 $\mathrm{Pa}$ (see Figure 1). In the literature, investigators typically attribute these differences to methodological differences, implying that brain tissue in itself cannot exhibit such a wide dispersion of stiffness values. Such hypothetical 'superviscous' behavior of brain tissue has never been observed experimentally in vivo. For brain tissue in general there are a number of possible reasons: first, most test methods cannot address low and high dynamic stimulations with the same precision $\underline{25}$. Second, many ex vivo methods are local, i.e., they test smaller (solid) tissue regions and ignore effects of fluid compartments although they are potentially important for the viscous properties $\underline{42}$. Finally, only MRE can 
measure stiffness of bulky tissue such as the brain at different frequencies in vivo and without tissue destruction or invasive procedures to the skull 24. However, MRE typically exploits a mechanical frequency range of 30 to $100 \mathrm{~Hz} \underline{43}$ leaving a significant gap of values to quasi-static ex vivo methods.

In fact, viscoelastic tissue properties measured at ultra-low stimulation frequencies below $20 \mathrm{~Hz}$ are widely unexplored in vivo 29.44 . The reason is related to long wavelengths exceeding the size of the brain and causing instabilities in inverse problem solutions. Nevertheless, we hypothesize that measurement of large-scale brain properties is possible even at ultra-low excitation frequencies of $5 \mathrm{~Hz}$ when analyzing global wavelengths across full hemispheres including all interfaces, heterogeneities, vessels, and fluid compartments. Exploiting intrinsic actuation by arterial pulsation as proposed by Weaver et al. might additionally open a window into $1 \mathrm{~Hz}$ (harmonic) frequency MRE $\underline{45}, \underline{46}$.

Combining intrinsic actuation with ultralow-frequency MRE requires a new way of displacement sampling, preferably in real time. Therefore, we here use novel steady-state MRE with spiral readout and stroboscopic undersampling of harmonic vibrations (sSMRE)느. Furthermore, we developed single-shot ssMRE to capture endogenous shear wave components in real time without synchronization to the cerebral pulse wave.

Uniquely, the combination of intrinsic and extrinsic ssMRE allows us to quantify in vivo human brain stiffness across more than 5 octaves of frequencies from 1 to $40 \mathrm{~Hz}$ in order to bridge, for the first time, two distinct ranges of brain stiffness: (i) supersoft properties as illustrated in Figure 1 and observed by Budday et al.41 using quasi-static tests in fresh post mortem human brain and (ii) the stiffness range encountered in clinical brain examinations using in vivo MRE. Collectively, we aim at establishing ground-truth values of in vivo brain stiffness at ultra-low dynamic deformations as relevant for ex vivo tests, surgical interventions, modeling $\underline{48}$, biomaterial engineering $\underline{49}, \underline{50}$ and potentially as a new cerebral MRE technique.

Finally, the range of shear moduli presented in this paper went beyond that reported in the literature (though only at frequencies currently unexplored). We performed an ex vivo calf brain experiment and measured the shear modulus via inverse finite element 
techniques. These results lend credibility to our reported low frequency shear moduli range.

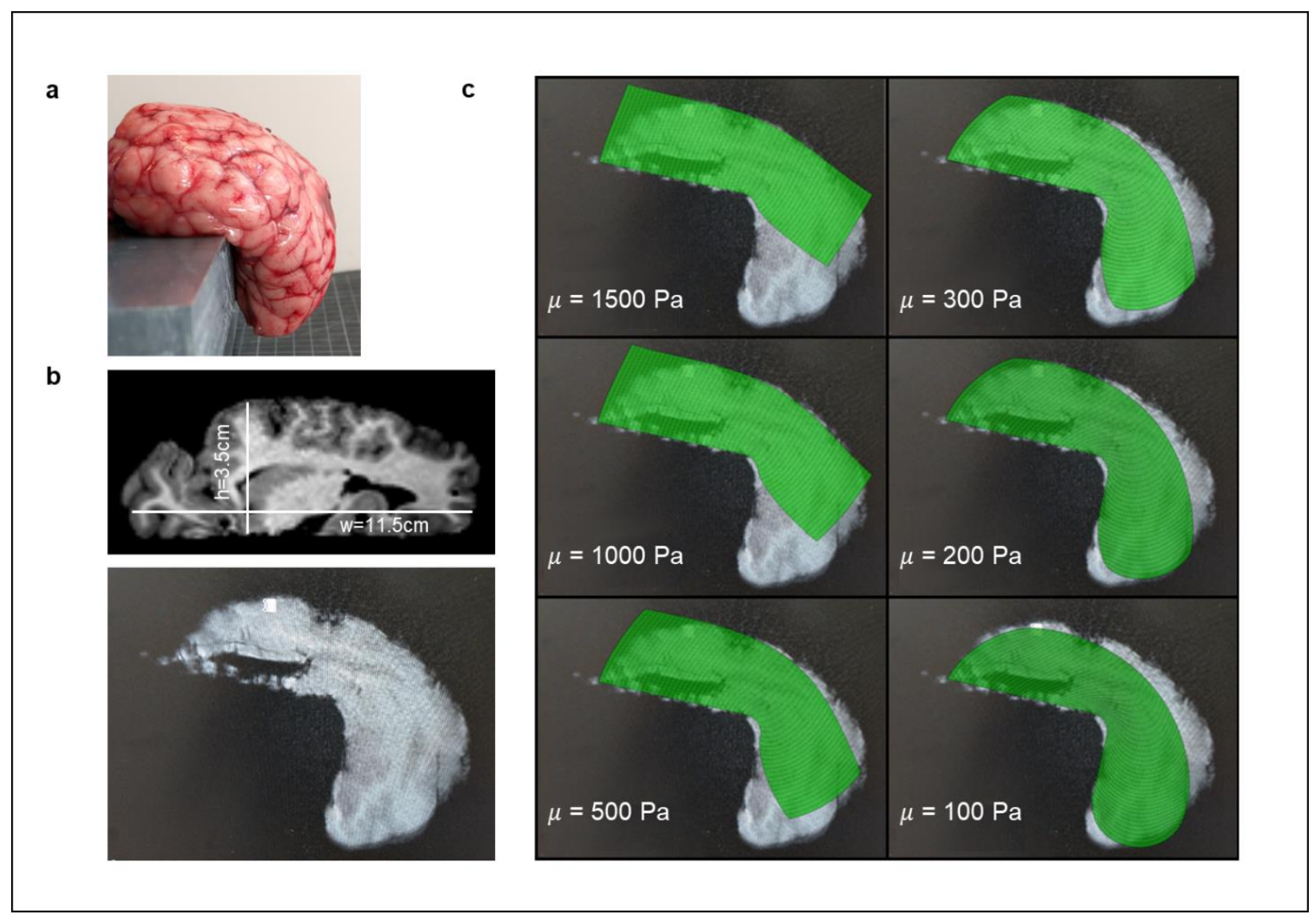

Figure 1: Brain tissue is super soft, as simple bending experiments on fresh calf brain show. a) Representative example of fresh brain hanging over an edge and bent by gravity. b) Gravity induced deformation can be visualized by MRI for later quantitative analysis. c) FEM simulations with different shear moduli to reproduce the brain deformation in a sagittal view.

\section{Methods}

\section{Subjects}

In vivo MRE was performed in 14 healthy volunteers without a history of neurological diseases ( 3 females, mean age \pm SD: $30 \pm 5$ years, age range: 24 to 44 years). The study was approved by the ethics committee of Charité - Universitätsmedizin Berlin in 
accordance with the Ethical Principles for Medical Research Involving Human Subjects of the World Medical Association Declaration of Helsinki. Every participant gave written informed consent.

\section{Ex vivo calf brain experiment}

The ex vivo experiment was performed on a fresh brain from a 22 weeks old calf obtained from a local butcher. The brain was transported in a $0.9 \%$ saline solution in a cooling box at approximately $4^{\circ} \mathrm{C}$ to prevent dehydration and tissue degeneration. The brain was photographed to portray the setup in the scanner and then placed in a 3T MRI scanner (Siemens Lumina, Erlangen, Germany). A three-dimensional magnetization-prepared rapid gradient-echo imaging (3D MP RAGE) with $1 \times 1 \times 1 \mathrm{~mm}^{3}$ voxel size was used to measure the brain in two different states (Figure 1b.). First, an undeformed state was examined for an anatomical reference image. Afterwards the brain was fixed on a holder in such a way that it would naturally bend under gravitation over the edge perpendicular to its longitudinal axis (anterior-posterior) as depicted in Figure 1a. These two anatomical scans were used for further FEM simulations as explained below.

\section{FEM simulations}

To estimate the stiffness of the calf brain, a finite element model of the brain was constructed in ABAQUS FE software (ABAQUS 2019, Dassault Systèmes Simulia Corp., Providence, RI, USA) to best match the brain configuration when subject to gravity loading as described in the ex-vixo calf brain experimental set up. The brain model consisted of two parts; homogenous brain tissue representing combined stiffness of white and grey matter, and the pia mater, a stiff membrane surrounding this tissue. A slice of the brain was modelled under plane strain conditions through the thickest part of the brain. Idealizing the brain slice as a rectangle, the slice geometry was based on the water bath rest geometry with a height of $3.5 \mathrm{~cm}$ and length of $11.5 \mathrm{~cm}$ (see Figure 1b) while the brain's top and end curvature was ignored. Given the water content of the brain, the elements of the brain tissue were considered incompressible and meshed with 6,962 C3D8H elements consisting of 10,800 nodes. The pia mater membrane was modelled with M3D4 membrane elements surrounding the outer brain tissue elements with a 
thickness of $15 \mu \mathrm{m} 5152$. Boundary conditions were set to encaster the nodes on the bottom of the slice which touched the support as no sliding was observed. To ensure plane strain, the walls of the slice were set with a fixed-in-plane condition. The mechanical properties of the brain tissue were modelled as a hyperelastic Neo-Hookean material with unknown stiffness. The pia mater was also considered as a Neo-Hookean material with a hyperelastic constant $C_{10}$ of $858.33 \mathrm{kPa}$ based on a Young's modulus value of 5.15 $\mathrm{MPa}$ which is an average value of the pia mater 51,53 . It is not worthy that hyperelasticity consideration for both tissues allow accounting for both intrinsic and geometric nonlinearities anticipated to be encountered in the experiment. To capture the bending of the brain tissue under gravity, the slice was loaded with its own weight; tissue density was assigned as $1081 \mathrm{~kg} / \mathrm{m}^{3}$ and gravity set to $9.806 \mathrm{~m} / \mathrm{s}^{2} \underline{\underline{4}}$.

Given the complexity of modelling soft tissue where self folding is a common occurrence leading to convergence issues; the slice was constrained to bend about a pre-set radius of $0.75 \mathrm{~cm}$ at a $7 \mathrm{~cm}$ bending location measured from the imaging session. A rigid contact wall was installed to keep any part of the tissue from bending beyond the turning point, but still allowed the tissue to fall with no friction.

To estimate the initial shear modulus, $\mu$, of the brain tissue which is equal to $2 C_{10}$, two criteria of the bending model had to be met according to experimental observations. First, the material must bend under its own weight to $90^{\circ}$, and second, the material must extend to $4.5 \mathrm{~cm}$ based on the image data. The model was run with variable initial shear modulus values starting from $1500 \mathrm{~Pa}$ with step increments of $50 \mathrm{~Pa}$, down to $50 \mathrm{~Pa}$ until both conditions were met.

\section{Phantom}

Before the in vivo study, phantom tests were performed in heparin-sodium gel (180 000

IU per $100 \mathrm{~g}$, Ratiopharm, Ulm, Germany) to validate fitting of complex exponential functions to 1D wave propagation profiles as explained below. The gel mainly consists of Carbomer 980 (polyacrylic acid), trometamol (TRIS), glycerol hydroxystearate, propylene glycol, and isopropanol with the eponymous active agent only accounting for less than 1 
$\%$ of the gel volume. MRE experiments were performed using the acquisition protocol of in vivo ssMRE.

\section{Oscillatory shear rheometry}

A shear rheometer (MCR301, Anton Paar, Graz, Austria) was used to measure a cylindrical sample from the same heparin gel as used in the MRE experiments. The sample had a diameter of $50 \mathrm{~mm}$ with a fixed plate-to-plate gap of $1 \mathrm{~mm}$. The linear range of viscoelasticity over strain amplitude was determined with amplitude sweeps at vibration frequencies of $5 \mathrm{~Hz}$ and $40 \mathrm{~Hz}$. Linear behavior of the material was still observed at $3 \%$ strain amplitude of both frequencies so that we chose this value for measuring complex shear modulus $\left|\mathrm{G}^{*}\right|$ at ten frequencies from $5 \mathrm{~Hz}$ to $40 \mathrm{~Hz}$. The experiments were repeated 12 times to obtain margins of measurement variability. Sample temperature was kept constant at $22^{\circ} \mathrm{C}$.

\section{ssMRE experimental setup}

All experiments were performed in a 1.5T MRI scanner (Siemens Magnetom Sonata, Erlangen, Germany). Three scans were consecutively acquired in each volunteer:

(I) endogenously activated cerebral SsMRE without external wave stimulation;

(II) ultra-low frequency time-harmonic SSMRE with external wave stimulation at frequencies $5,6.25,7.8125$, and $10 \mathrm{~Hz}$ frequencies;

(III) ssMRE with externally induced waves in a conventional higher frequency range of $20,31.25$, and $40 \mathrm{~Hz}$.

(II) and (III) were also performed in the phantom. External harmonic vibrations were introduced by pressurized air drivers as described elsewhere 47 . The vibrations were induced with a forerun of $2 \mathrm{~s}$ before MRE data acquisition was started in order to establish a steady state of time-harmonic oscillations throughout the phantom or brain. The wave generator was synchronized with the MRI clock to avoid any latency between MRE data acquisition and wave generation $\underline{5 .}$. The output frequencies were automatically switched 
by a 'smart trigger' as explained in $\underline{56}$. Given the nonlinear motion characteristics of the actuators at low vibration frequencies, a certain amount of acoustic wave energy was transmitted into the brain through higher harmonics of the fundamental driving frequency. Therefore, we could analyze the second harmonic $(12.5 \mathrm{~Hz})$ of $6.25 \mathrm{~Hz}$, the second $(15.625 \mathrm{~Hz})$ and third $(23.4375 \mathrm{~Hz})$ harmonics of $7.8125 \mathrm{~Hz}$, and the third harmonic (30 $\mathrm{Hz}$ ) of $10 \mathrm{~Hz}$, while we did not use other higher harmonics due to either low amplitudes or redundant frequencies.

\section{SSMRE sequence}

The principle of stroboscopic undersampling of harmonic vibrations was implemented in a steady-state gradient-echo MRE sequence with spiral readout as detailed in. Synchronized stroboscopic wave sampling (ssMRE47) allows higher frame-rates than what would be possible with serial imaging, when the Nyquist sampling limit is determined by the frame rate of the MRE sequence $\underline{47}$.

For (I), intrinsic activation ssMRE, a single-shot version of sSMRE was employed to acquire a sequence of 334 2D single-slice, spiral k-spaces with a sampling rate of 16.7 $\mathrm{Hz}$ over a total of $20.04 \mathrm{~s}$ similar to the method described in $\underline{57}$. The sequence was started using a cardiac trigger to ensure acquisition of identical phases of wave components relative to the cerebral arterial systole. All three components of the wave field were consecutively acquired (see Figure 2). Further sequence parameters were: repetition time $(\mathrm{TR})=60 \mathrm{~ms}$, echo time $(\mathrm{TE})=19.9 \mathrm{~ms}$, motion-encoding gradient amplitude $(\mathrm{MEG})=$ $30 \mathrm{mT} / \mathrm{m}$ (zero-moment nulling), field of view $(F o V)=220 \times 220 \mathrm{~mm}^{2}, 1.7 \times 1.7 \times 5 \mathrm{~mm}^{3}$ voxel size, total scan time $=126 \mathrm{~s}$.

For (II), ultra-low frequency ssMRE, the same sequence as in (I) was used but with segmentation of each k-space into nine spiral trajectories to reach higher resolution. Therefore, each k-space trajectory was sampled 222 times at a sampling rate of $22.2 \mathrm{~Hz}$ over $9.99 \mathrm{~s}$, followed by sampling of the trajectories of the next k-space. Again, all three components of the wave field were consecutively acquired. Four such full ssMRE experiments were run at distinct vibration frequencies of $5,6.25,7.8125$, and $10 \mathrm{~Hz}$. 
Further sequence parameters were: $\mathrm{TR}=45 \mathrm{~ms}, \mathrm{TE}=20 \mathrm{~ms}, \mathrm{MEG}=30 \mathrm{mT} / \mathrm{m}$ (zeromoment nulling), FoV $=220 \times 220 \mathrm{~mm}^{2}, 1.1 \times 1.1 \times 5 \mathrm{~mm}^{3}$ voxel size, total scan time $=$ $276 \mathrm{~s}$.

For (III), conventional-frequency ssMRE at $20,31.25$, and $40 \mathrm{~Hz}$ vibration frequency, the same protocol as in (II) was used but with $\mathrm{TR}=40 \mathrm{~ms}$ and TE $=20 \mathrm{~ms}$, resulting in a frame rate of $25 \mathrm{~Hz}$ and scan time of $8.88 \mathrm{~s}$ for each wave field component. Total scan time for (III) was $126 \mathrm{~s}$.

Data were acquired in a single transverse image slice at the level of the basal nuclei along the largest diameter of the lateral ventricles in the sagittal plane.

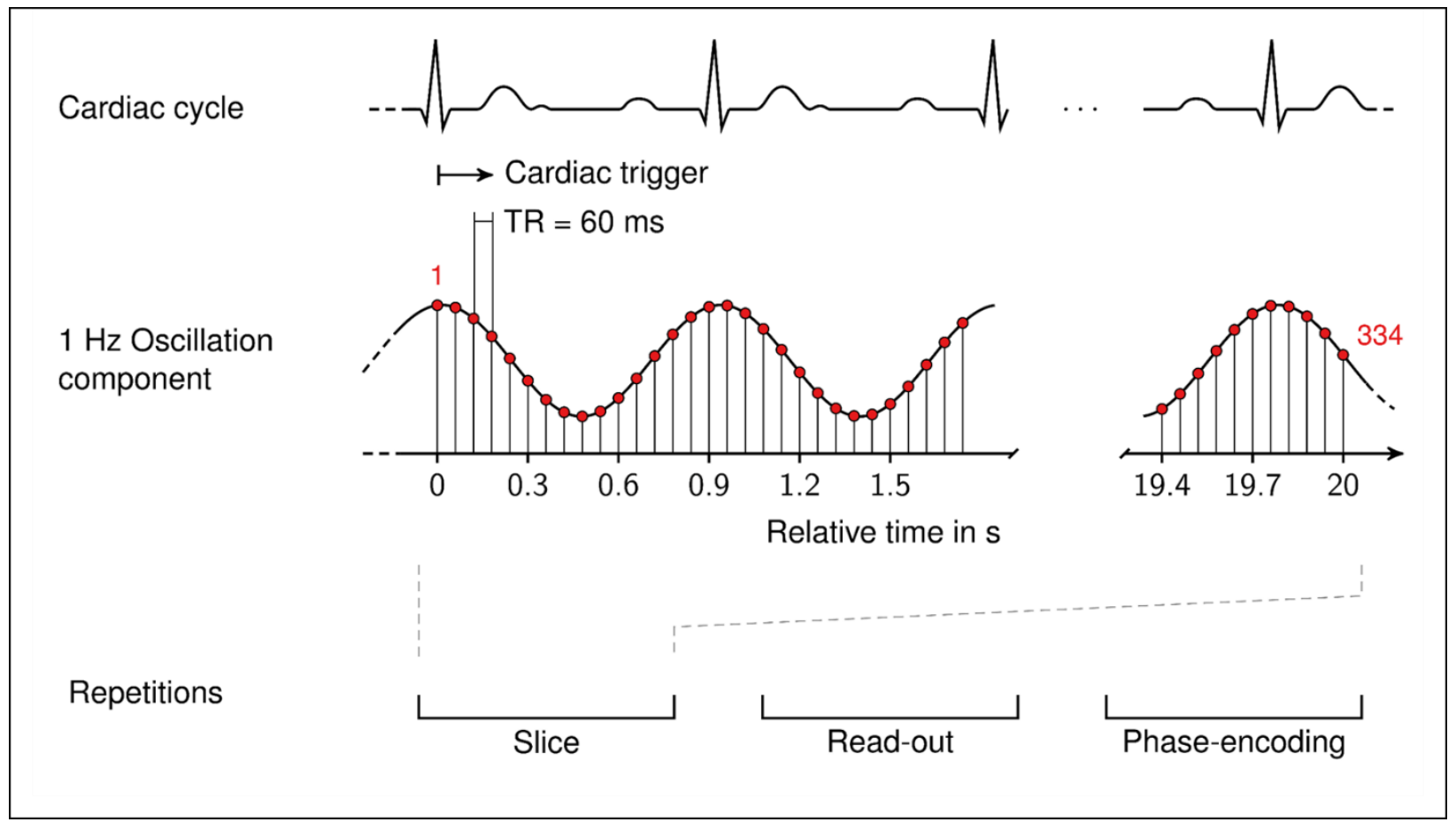

Figure 2: Echo timing diagram. Steady-state gradient echo timing diagram with spiral readout trajectory for intrinsic activation MRE (single shot).

Data analysis by wave profile fits

Reference shear wave speed (SWS) values (in $\mathrm{m} / \mathrm{s}$ ) were derived based on manually drawn 1D profiles perpendicular to the main direction of wave propagation for all frequencies. Externally induced shear waves cross the brain from multiple directions, 
predominantly along the left-to-right axis when the waves are polarized in the anteriorposterior direction. Henceforth, we picked profiles of this component along the left-to-right axis in both hemispheres to analyze wavenumbers by $1 \mathrm{D}$ least-square fitting. The fitting model comprised two damped plane waves propagating into opposed directions with identical complex wave numbers $k=k^{\prime}+i k^{\prime \prime}$, but different amplitudes $A_{1}$ and $A_{2}$ and initial phases $\varphi_{1}$ and $\varphi_{2}$ :

$$
u(x)=A_{1} \cdot e^{+i\left(k x+\varphi_{1}\right)}+A_{2} \cdot e^{-i\left(k x+\varphi_{2}\right)}
$$

A simplified model function comprises only a single propagating wave as given by:

$$
u(x)=A_{1} \cdot e^{+i\left(k x+\varphi_{1}\right)}
$$

Fitting of complex 1D wave profiles was performed in MATLAB (MathWorks Inc., Natick, MA, USA, Release 2019b) using the function fminsearch with equations (1) or (2) as model functions and $k, A_{1}, A_{2}$ and $\varphi_{1}$ and $\varphi_{2}$ as input variables.

Equation (2) is a uni-directional plane wave. Fitting MRE data with this model requires that the MRE wave field has also been decomposed into uni-directional waves. This is typically done by directional filtering in the Fourier domain However, Fourier transformation introduces spurious signals due to the limited size of the field of view (Gibbs ringing). At very low wavenumbers such ringing of k-space signals can cross the origin of k-space leading to portions of wave signal, which has, spuriously, opposite directionality. Consequently, very low positive (or negative) wavenumbers can leak into negative (or positive) spatial frequencies making directional filtering prone to bias. Figure 3 illustrates this bias by simulations showing that wavelengths are increasingly underestimated when input wavenumbers become too low.

Equation (1) offers a solution to this bias by taking into account that waves can travel bidirectionally along a profile. Thus, a directional filter is not needed here leaving wavenumbers widely unaffected and resulting in more stable values. Figure 3 shows that bias due to equation (1) is much lower than bias due to equation (2), latter combined with directional filters as shown in Figure 3. 
This strategy was validated by numerical simulations and phantom experiments. Of note, in vivo data acquired by intrinsic activation display a wave that emanates from the circle of Willis and, thus, is single-sourced. Nevertheless, also here we used equation (1) for fitting instead of the bi-directional wave model of equation (2) since numerical simulations indicated that equation (1) also matches unidirectional waves. Finally the profile-based wavenumbers were converted to SWS values.

\section{Model fitting}

The SWS dispersion curves obtained for the phantom and in vivo data were fitted using Kelvin-Voigt, Maxwell, spring-pot, and viscous models to identify the corresponding model parameters. The model functions of the complex shear modulus $G^{*}=G^{\prime}+i^{*} G^{\prime \prime}$ are presented in Table 1. The complex shear modulus can be converted into SWS on the basis of absolute value $\left|\mathrm{G}^{*}\right|$ and phase angle $\varphi$ or storage modulus $\mathrm{G}^{\prime}$ and loss modulus G" by:

$$
S W S=\sqrt{\frac{2 \cdot\left|G^{*}\right|}{\rho \cdot(1+\cos (\varphi))}}
$$

or

$$
S W S=\sqrt{\frac{2 \cdot\left(G^{\prime 2}+G^{\prime \prime 2}\right)}{\rho \cdot\left(G^{\prime}+\sqrt{G^{\prime 2}+G^{\prime 2}}\right)}} .
$$

$\rho$ denotes tissue density and was set to $1 \mathrm{~kg} / \mathrm{m}^{3}$. A least-squares algorithm was used to estimate the model parameters expected to minimize the deviations of the theoretical curve from the experimental curve including the standard deviation of each measurement point as a weighting factor $\underline{58}$. The standard errors of the measured parameters were calculated including partial derivatives of the fitted curve with the propagation of errors and the standard deviations of the measurement points included. 


\section{Statistical tests}

Nine healthy volunteers were investigated a second time to assess the repeatability of in vivo ssMRE. This was analyzed by (i) coefficient of repeatability $\left(\mathrm{C}_{\mathrm{R}}\right) \underline{\underline{9}}$, as well as (ii) relative absolute difference $\left(R A D_{i}\right)$ between corresponding measurements for each subject $j$ from a total of $n$ subjects. These parameters are defined by:

$$
\begin{aligned}
& C_{R}=1.96 \cdot \sqrt{\sum_{j=1}^{n} \frac{\left(\overline{c_{1, j}}-\overline{c_{2, j}}\right)^{2}}{n}} \\
& R A D_{i}=\frac{2 \cdot\left|\overline{c_{1, j}}-\overline{c_{2, j}}\right|}{\left(\overline{c_{1, j}}+\overline{c_{2, J}}\right)}
\end{aligned}
$$

$\overline{c_{1, j}}$ and $\overline{c_{2, J}}$ represent SWS values derived from profile-fits of subject $j$ for measurements 1 and 2.

\section{Results}

\section{FEM simulations}

Figure 1 illustrates the low flexural modulus of brain tissue at large scales. It is well visible that brain tissue, which is statically deformed by gravity, cannot hold its own weight. The simulations overlaid with brain MRI indicate a bending shear modulus on the order of 100 $\mathrm{Pa}$. Measurement of a similar mechanical response, but in vivo, requires MRE to be combined with ultra-low vibration frequencies - far below the standard range of excitation frequencies. To determine the limits of large-scale wavelength analysis, numerical simulations are presented in the following.

\section{Validation of large-scale wavelength analysis by numerical simulations}

Representative 1D scenarios demonstrating the effect of directional filtering in the Fourier domain at high $(360 / \mathrm{m})$ and low $(26 / \mathrm{m})$ wave numbers (50 sample points, added 
Gaussian noise, $\sigma=0.03$ ) are presented in Figure 3 . The presence of artifacts caused by suppression of residuals of the wanted (positive) signal within the negative half of k-space (Figure 3c) is indicated by deformed waves (Figure 3a and 3b). The affected curvature of the waves results in underestimated wave numbers when fitting is performed by equation (1). Figure $3 \mathrm{~d}$ shows a systematic bias of less than approximately 1.2 wavelengths are covered by the profile as input data.

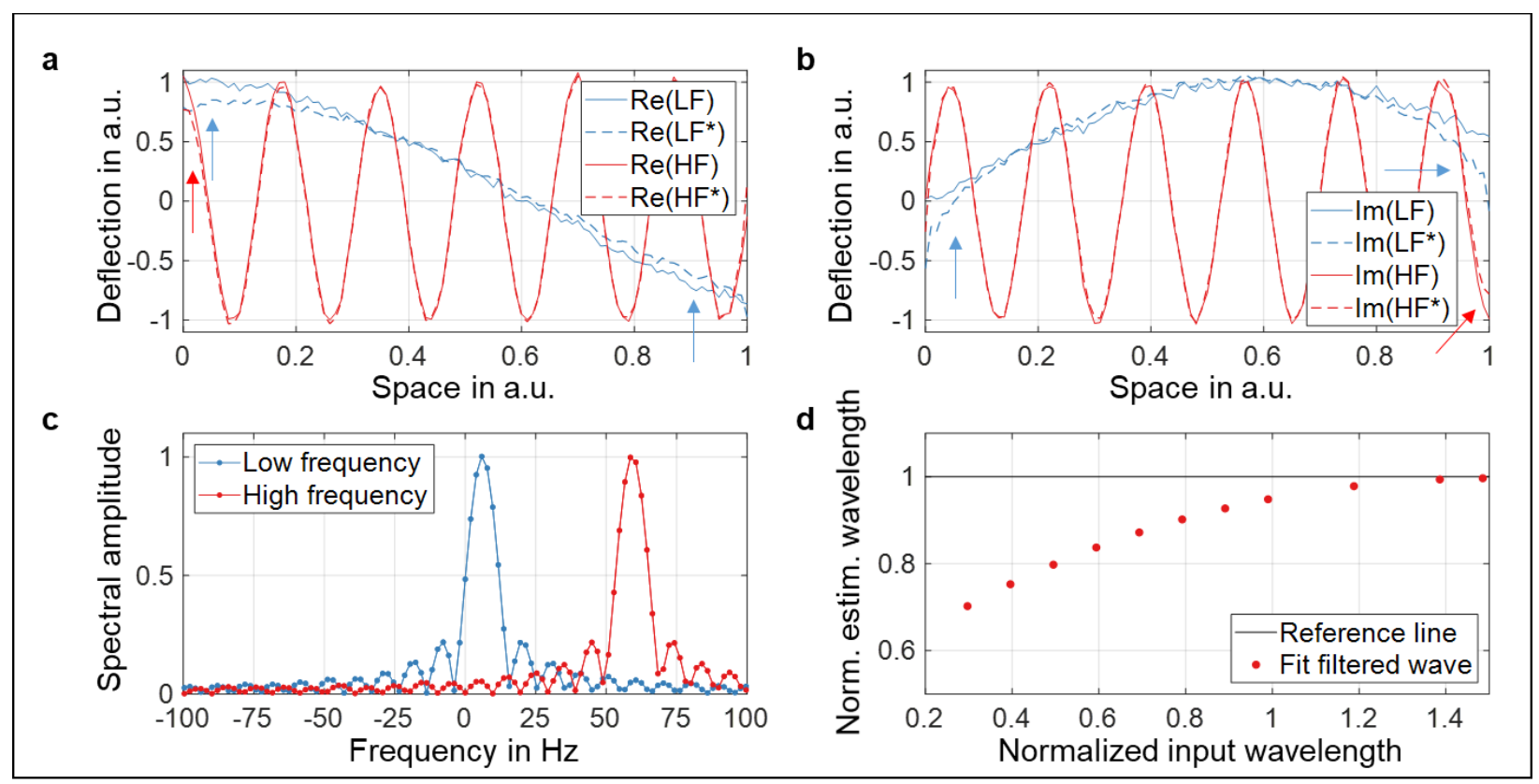

Figure 3: Numerical simulation demonstrating the impact of directional filtering, i.e. suppression of negative frequencies, on 1D complex waves with different wavelengths. a) Real and b) imaginary parts of the complex waves before and after applying directional filters. Deformed waves (arrows) are due to suppression of all negative k-space frequencies. c) Ringing-related spectral amplitudes in the negative Fourier domain of both unfiltered waves are typically suppressed when directional filters are applied yielding the bias in the wave curvature shown in (a) and (b). d) Numerical simulation of 1D complex wave fit after directional filter and recovery of wavelength for different input wavelengths.

Figure 4 presents the errors expected for two different scenarios, 1D uni- and bidirectional waves, each fitted according to equation (1) or equation (2). The reference wavelength was normalized to 1 with added Gaussian noise $\sigma=0.01$. Interestingly, results varied little 
with noise but changed significantly when initial phases $\left(\varphi_{1}\right.$ and $\left.\varphi_{2}\right)$ were cycled from 0 to $2 \pi$. Hence, the error bars, indicating standard deviations of repeated simulations, mainly reflect variability due to $\varphi_{1}$ and $\varphi_{2}$ phase variations. It is well visible that, for unidirectional input waves, the corresponding unidirectional wave fit according to equation (1) yields more reliable estimates at low wavelengths (10\% error at 0.14 wavelengths) than the bidirectional wave fit of equation (2) (10\% error at 0.25 wavelengths).

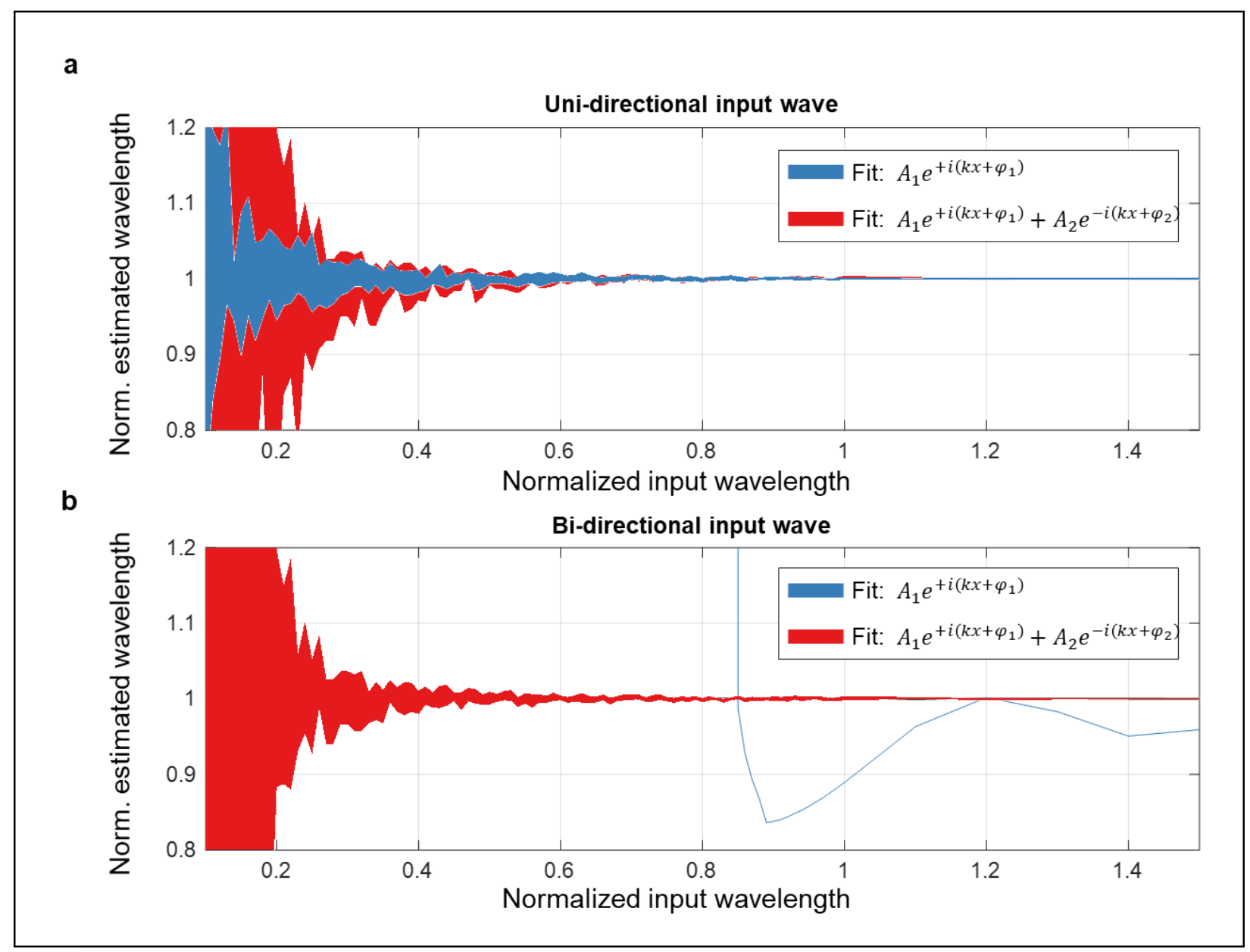

Figure 4: Numerical simulation of different models for the fitting routine when smaller fractions of a total wavelength are used as input data. Two scenarios are shown: bidirectional and unidirectional waves propagating along 1D profiles according to equations (1) and (2), respectively. Standard deviations indicate the variation obtained from repeated simulations with changing initial phases of the waves. 
However, analyzing bidirectional waves, equation (1) entirely fails at less than a full wavelength while equation (2) still provides acceptable results $(10 \%$ error at 0.25 wavelengths). This analysis indicates that wave profiles of $4.5 \mathrm{~cm}$ length permit analysis of extrinsic (bidirectional) waves of approximately $18 \mathrm{~cm}$ length using equation (2) and intrinsic (unidirectional) waves of $32 \mathrm{~cm}$ length using equation (1) with error margins of less than $10 \%$.

\section{Validation of large-scale wavelength analysis by phantom experiments}

Figure 5 shows SWS dispersion over frequency in a heparin phantom measured with shear rheometry in comparison to the fit of 1D profiles by equation (2) along the main propagation direction. As with in vivo data acquisition in the brain, SWS was recovered from the fundamental frequency and its higher harmonics. Viscoelastic model fitting to profile-based SWS data revealed that the spring-pot model better reproduced SWS dispersion in heparin phantoms than the other two-parameter models, in agreement with the results in60. Other than later shown in the brain, the viscous model did not match the experimental data and was thus omitted from the figure.

In vivo large-scale wavelength analysis

Evaluation of intrinsic MRE is presented in Figure 6. Wave images in a volunteer are displayed in Figure 6a. Snapshots of all three wave-field components are shown along with their frequency spectra obtained from temporal Fourier transformation over 334 wave images. The spectra in Figure $6 \mathrm{~b}$ were extracted at the level of the circle of Willis, from which the intrinsically activated pulse wave emanates with a major deflection component in the head-feet direction61. Due to the nonlinear nature of cerebral arterial pulsation, intrinsic MRE spectra reveal the presence of higher harmonics of the subject's heart rate. Furthermore, multiple peaks around $4 \mathrm{~Hz}$ are visible, which are probably caused by aliased system vibrations of the MRI scanner. In the case shown, wave images were taken from the 24th bin of the spectrum corresponding to $1.2 \mathrm{~Hz}$ fundamental frequency of arterial pulsation. Thus, the waves, which we later analyzed by 1D profile fitting 
represent $1.2 \mathrm{~Hz}$ harmonic motion despite the nonharmonic nature of pulsation-induced brain motion. For illustration, an estimate of pulse wave speed is shown in Figure $6 \mathrm{c}$ by manually delineating the gradient of the wave phase in $x$ - $t$ space.

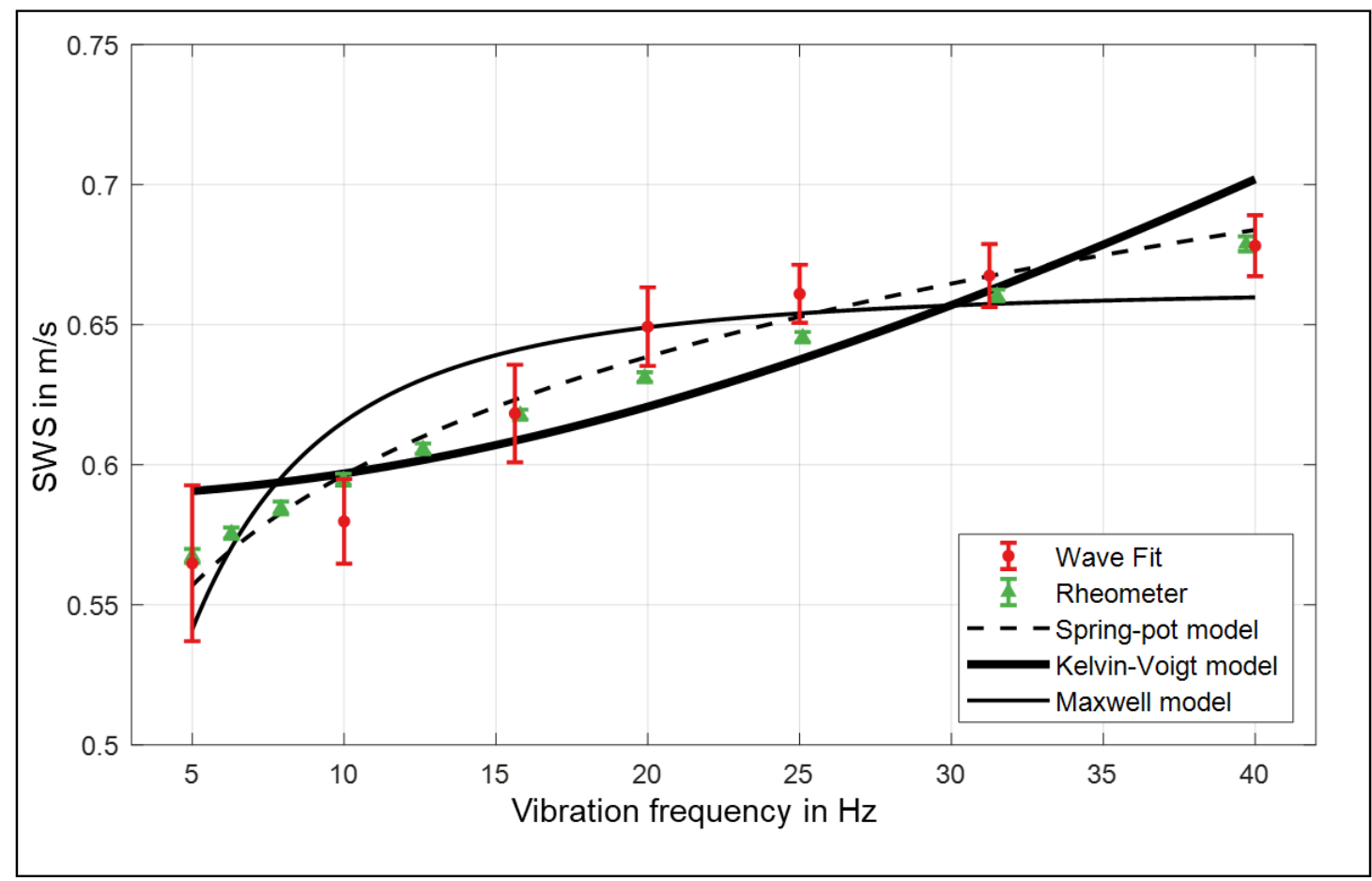

Figure 5: SWS of heparin gel phantom for different vibration frequencies $(5-40 \mathrm{~Hz})$. SWS was quantified by sSMRE and 1D profile-based fits using bi-directional plane waves as model function, equation (1). Ground-truth values were taken from shear oscillation rheometry. In addition, different rheological models are fitted to the profile-based sSMRE data.

Evaluation of external actuation ssMRE is presented in Figure 7. Wave images in a single volunteer over the range from intrinsic activation to $40 \mathrm{~Hz}$ are presented in Figure 7a. For intrinsic MRE, the harmonic wave field component at approximately $1.2 \mathrm{~Hz}$ in head-feet direction is shown whereas the other wave fields display the anterior-posterior encoding direction. Figure $7 \mathrm{~b}$ presents plots of 1D complex wave profiles from the location indicated by the green arrow above. Shorter wavelengths with higher frequencies are well visible. 


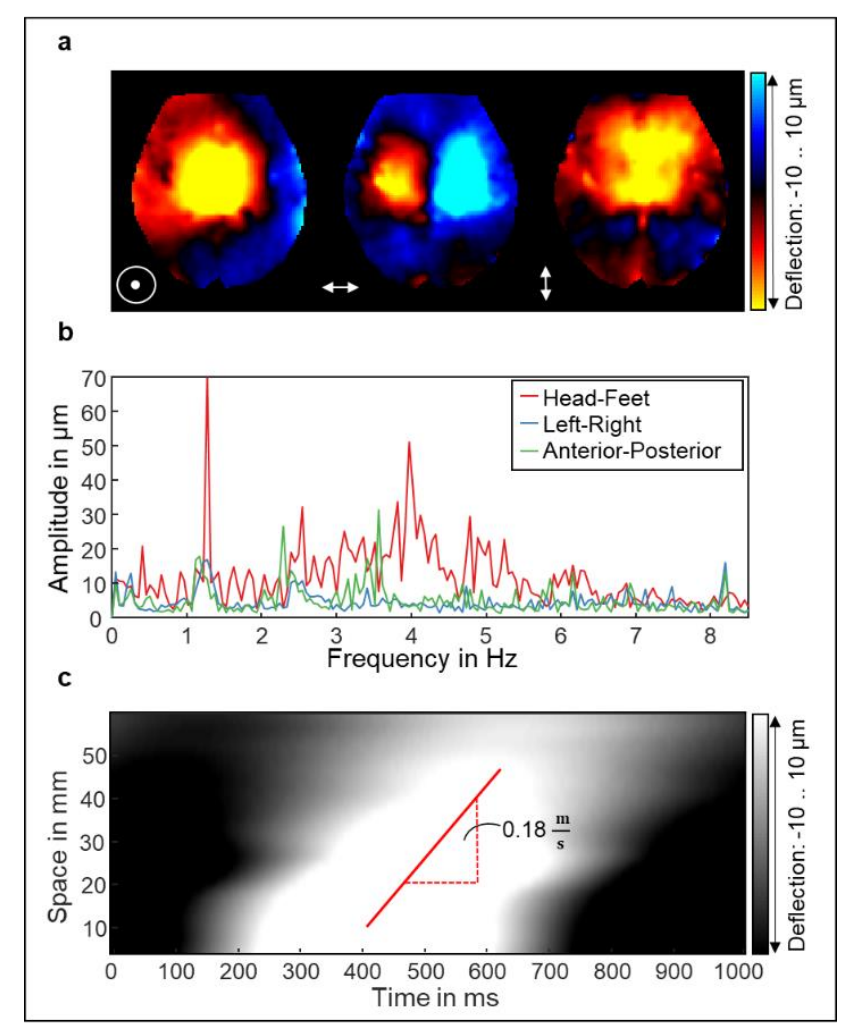

Figure 6: Analysis of intrinsically activated MRE. Shown are a) representative deflections of wave images for the three encoding components $(\odot, \leftrightarrow, \downarrow$ denote deflections through-plane [head-to-feet], left-right, and up-down [anterior-posterior], respectively) and b) Fourier spectrum of measured time series in the central region around major blood vessels in the circle of Willis for three deflection components. c) Space-time plot of propagating pulse wave with manual slope estimation for the SWS.

Averaged SWS values over frequency are plotted in Figure 8 and compiled in Table 2. For comparison, values reported by $\underline{37}$ for in vivo brain studies at 15,30 , and $45 \mathrm{~Hz}$ are shown, which were derived by three-frequency direct inversion and converted to SWS by equation (3). Similarly, values obtained by $\underline{44}$ for frequencies of $15,20,25,30,35,40$, and $50 \mathrm{~Hz}$ are shown. Testu et al. $\underline{44}$ applied advanced nonlinear inversion at single frequencies to a subset of data from Dittmann et al. $\underline{37}$ to obtain storage and loss moduli G' and G", which were then converted to SWS by equation (4). 


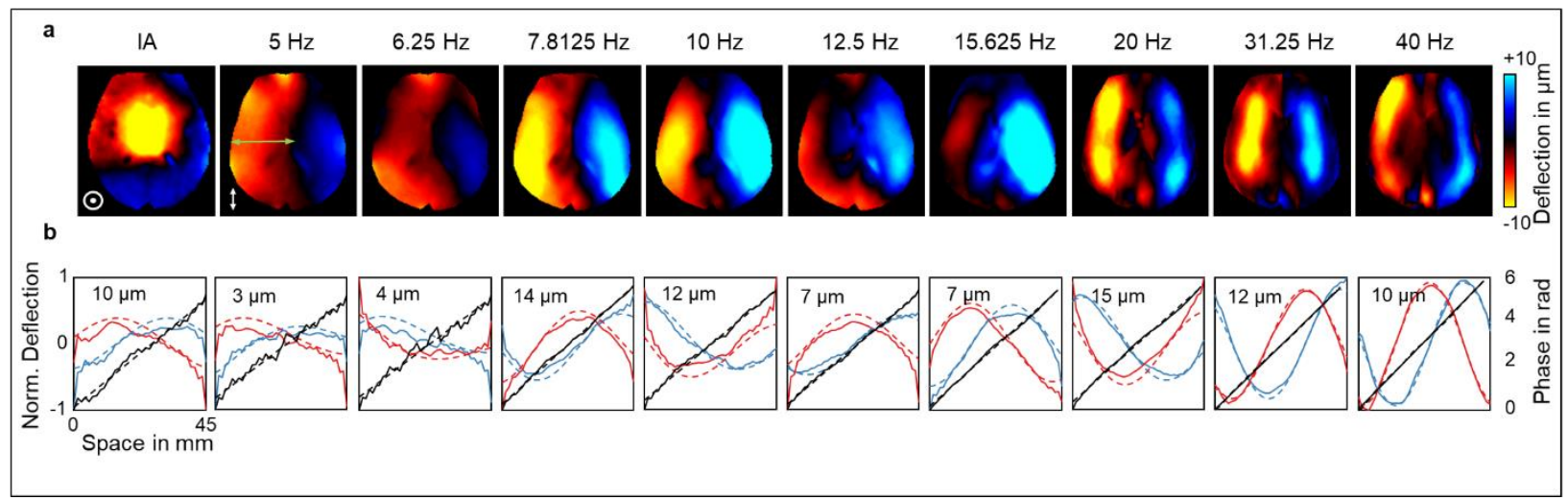

Figure 7: Analysis of ssMRE data. a) Representative wave fields with polarization in head-feet direction (first image) and anterior-posterior direction (rest of the images) for IA (intrinsic activation) and all driving frequencies (5-40 Hz). b) 1D fit (dotted lines) of bidirectional waves according to equation (1) to real (blue) and imaginary (red) parts of the complex wave profile taken from a position as demarcated by the arrow in the $5-\mathrm{Hz}$ image of (a). The phase is indicated by the black lines and the phase fit by a black dotted line.

Fitting of rheological models to profile-based results revealed that Maxwell and springpot models collapsed to the pure-fluid (viscous) model. Accordingly, shear modulus $\mu$ could not be determined for the Maxwell and spring-pot model could not be determined, while $\eta$ was $6.23 \pm 0.22 \mathrm{~Pa} \cdot \mathrm{s}$ based on the viscous model (see Table 1 ). The super viscous nature of brain tissue was reflected by very low $\mu$ and relatively high $\eta$ values using the standard two-parameter solid model (Kelvin-Voigt model, $\mu=42 \pm 13 \mathrm{~Pa}, \eta=6.57 \pm 0.30 \mathrm{~Pa} \cdot \mathrm{s}$ ).

\section{Reproducibility}

We analyzed reproducibility of ssMRE at, so far, unexplored frequencies of 5 and $10 \mathrm{~Hz}$. Coefficient of reproducibility $C_{R}$ was $0.07 \mathrm{~m} / \mathrm{s}$ at $5 \mathrm{~Hz}$ and $0.06 \mathrm{~m} / \mathrm{s}$ at $10 \mathrm{~Hz}$ with mean values of $0.52 \pm 0.06 \mathrm{~m} / \mathrm{s}$ and $0.79 \pm 0.03 \mathrm{~m} / \mathrm{s}$, respectively. Relative absolute difference, $R A D_{i}$, was $6.6 \%$ (mean) and $8.9 \%$ (maximum) for $5 \mathrm{~Hz}$ and $2.8 \%$ (mean) and $7.8 \%$ (maximum) for $10 \mathrm{~Hz}$. 


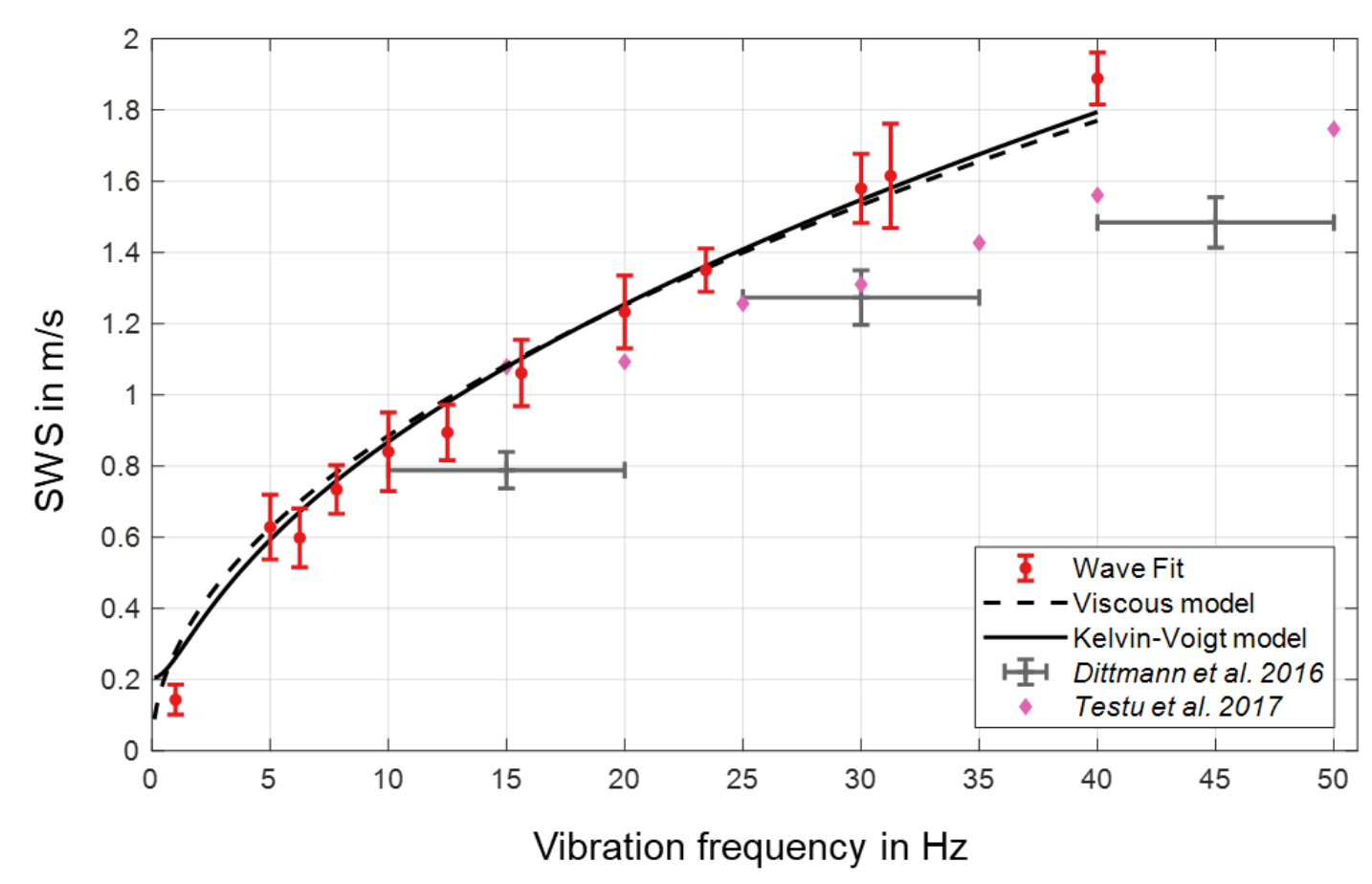

Figure 8: Dispersion curve of SWS in the human brain obtained by profile-based fitting routine for every frequency from manually depicted lines including intrinsic activation ssMRE. For comparison, values of Dittmann et al. $\underline{37}$ and Testu et al. $\underline{44}$ are shown. Two rheological models, the viscous model and the Kelvin-Voigt model (see Table 1), were fitted to the SWS data obtained by 1D profile fitting.

\begin{tabular}{|c|c|c|c|c|c|c|}
\hline \multirow[b]{2}{*}{ Model } & \multirow[b]{2}{*}{ Formula } & \multicolumn{2}{|c|}{ In vivo } & \multicolumn{3}{|c|}{ Phantom } \\
\hline & & $\eta$ in $\mathrm{Pa} \cdot \mathrm{s}$ & $\mu$ in $\mathrm{Pa}$ & $\alpha$ & $\eta$ in $\mathrm{Pa} \cdot \mathrm{s}$ & $\mu$ in $\mathrm{Pa}$ \\
\hline Spring-Pot & $G^{*}=\mu^{1-\alpha}(i \omega \eta)^{\alpha}$ & \multicolumn{2}{|c|}{ converges to viscous } & $0.20(0.02)$ & 1 (fixed) & $528(20)$ \\
\hline Maxwell & $G^{*}=\frac{\mu \cdot i \omega \eta}{\mu+i \omega \eta}$ & \multicolumn{2}{|c|}{ converges to viscous } & - & $8.06(1.18)$ & $440(15)$ \\
\hline Viscous & $G^{*}=i \omega \eta$ & $6.23(0.22)$ & - & - & $1.44(0.24)$ & - \\
\hline Kelvin-Voigt & $G^{*}=\mu+i \omega \eta$ & $6.57(0.30)$ & $42(13)$ & - & $1.08(0.11)$ & $346(15)$ \\
\hline
\end{tabular}

Table 1: Fit parameters (uncertainties given in brackets) of rheological models with corresponding model equations used to analyze the SWS dispersion curve obtained by profile-based sSMRE in human brain and in a heparin gel phantom. 


\begin{tabular}{cccccccccccccc}
\hline $\begin{array}{c}\text { Frequency } \\
\text { in Hz }\end{array}$ & 1 & 5 & 6.25 & 7.8125 & 10 & $\begin{array}{c}12.5 \\
(6.25)\end{array}$ & $\begin{array}{c}15.625 \\
(7.8125)\end{array}$ & $\begin{array}{c}20 \\
(10)\end{array}$ & 20 & $\begin{array}{c}23.4375 \\
(7.8125)\end{array}$ & $\begin{array}{c}30 \\
(10)\end{array}$ & 31.25 & 40 \\
\hline $\begin{array}{c}\text { Profile fit } \\
\text { SWS: }\end{array}$ & $\begin{array}{c}0.14 \\
\text { mean (SD) }\end{array}$ & $\begin{array}{c}0.04) \\
(0.09)\end{array}$ & $\begin{array}{c}0.60 \\
(0.08)\end{array}$ & $\begin{array}{c}0.73 \\
(0.07)\end{array}$ & $\begin{array}{c}0.84 \\
(0.11)\end{array}$ & $\begin{array}{c}0.89 \\
(0.08)\end{array}$ & $\begin{array}{c}1.06 \\
(0.09)\end{array}$ & $\begin{array}{c}1.22 \\
(0.10)\end{array}$ & $\begin{array}{c}1.24 \\
(0.07)\end{array}$ & $\begin{array}{c}1.35 \\
(0.06)\end{array}$ & $\begin{array}{c}1.58 \\
(0.10)\end{array}$ & $\begin{array}{c}1.62 \\
(0.14)\end{array}$ & $\begin{array}{c}1.89 \\
(0.07)\end{array}$ \\
\hline
\end{tabular}

Table 2: SWS (standard deviation given in brackets) measured by in vivo sSMRE in the brains of 14 healthy volunteers with profile-based estimation of SWS values.

\section{Discussion}

To our knowledge, this is the first study of in vivo MRE of the human brain investigating a wide frequency range from intrinsic pulsation at heart rate to $40 \mathrm{~Hz}$ external stimulation. Combining wave profile analysis for extrinsic and intrinsic MRE allowed us to measure the continuous increase in SWS of in vivo brain from 0.1 to $1.9 \mathrm{~m} / \mathrm{s}$. None of the two methods alone, intrinsic or extrinsic MRE, could have revealed the superviscous behavior of brain tissue, which is characterized by a large dispersion of stiffness values from $10 \mathrm{~Pa}$ to $3.6 \mathrm{kPa}$ over more than two orders of magnitude.

Our results show good reproducibility of SsMRE values for 5 and $10 \mathrm{~Hz}$, which are both outside the range that has been explored in vivo so far. Previous work on in vivo wideband MRE of the human brain has demonstrated the technical feasibility of using $10 \mathrm{~Hz}$ as drive frequency in MRE while data consistency at that specific frequency was lower than at higher frequencies37,44. McGarry et al. studied viscoelastic and poroelastic models at $50 \mathrm{~Hz}$ and $1 \mathrm{~Hz}$ brain simulation and demonstrated that mechanical property estimation using viscoelastic inversion performed poorly at low frequencies $\underline{29}$. Viscoelastic inversion techniques at $1 \mathrm{~Hz}$ have issues with non-unique solutions due to the small wave curvature relative to noise 62 . 63 . This is probably the reason why our results are different from results obtained with intrinsic activation MRE of the brain $\underline{45}$ at $1 \mathrm{~Hz}$, which are on the order of $8.1 \mathrm{kPa}$ for linear viscoelastic reconstruction and $2.4 \mathrm{kPa}$ for poroelastic reconstruction. This is clearly higher than expected from studies of fresh ex vivo human brain specimens reported by Budday et al. who proposed the term supersoft properties or as illustrated by our analysis illustrated in Figure 1. The speed of the propagating shear wave emanating from larger arteries in the circle of Willis can be visually noted in the x-t plots as shown in 
Figure 6a and provided as three separate animations in the supplementary material. The slope of the propagating wave clearly indicates a wave speed of approximately $0.18 \mathrm{~m} / \mathrm{s}$, corresponding to $32 \mathrm{~Pa}$ modulus according to the elastic model. Of note, possible misplacement of image plane and wave profile relative to the direction of wave propagation would have caused an overestimation of that SWS value, which further demonstrates the extremely soft properties of the human brain at this low dynamic stimulation frequency 64 . Furthermore, it should be mentioned that our model for fitting propagating waves incorporates an offset term which separates compression waves from the desired shear wave.

As analyzed in previous work, compression waves induced by cerebral arterial pulsation traverse the brain too fast to be tracked within an $x-t$ space $\underline{47}$. By contrast, arterial pulsation induces a shear wave with a speed that depends on the distensibility of the vessel walls. The pulsation-driven shear wave is strongly decelerated from $0.4 \mathrm{~m} / \mathrm{s}$ (in proximal segments of the middle cerebral artery $\underline{65}$ ) to very small values of $0.3 \mathrm{~mm} / \mathrm{s}$ in the neocortical capillaries of the mouse brain66. In white matter, the shear wave speed induced by pulsation of perforating arteries has been reported to be $0.5-1.0 \mathrm{~cm} / \mathrm{s} \underline{65}$. The value of $0.14 \mathrm{~m} / \mathrm{s}$ we found seems to average pulsation velocities across the full range of arterial diameters present in the brain, thus providing a measure of the effective medium properties of the brain including solid tissue and fluid compartments on large scales.

It is an intriguing result of our study that SWS measured with intrinsic pulsation is consistent with data obtained by extrinsic actuation when we consider the superviscous behavior of the brain. Our viscosity of $\eta=6.6 \pm 0.3 \mathrm{~Pa} \cdot \mathrm{s}$ is markedly larger than previously reported in the narrower frequency band of 25 to $62.5 \mathrm{~Hz}$ based on the same Kelvin-Voigt model $(2.1 \pm 0.4 \mathrm{~Pa} \cdot \mathrm{s})$ but agrees with the assumption of fluid-like brain properties by Bilston and colleagues $\underline{26}$. The observed superviscous behavior of in vivo brain might be explained by an abundance of fluid-filled vessels and pores $45,61,67,68$. Although the order of our very low shear modulus points towards reported values of supersoft properties, we attribute remaining differences to mixed effects of vascular distensibility, interstitial fluid motion, and post mortem stiffening $\underline{69} \cdot \underline{70}$ on the effective-medium viscoelastic properties at large scales $\underline{68}, \underline{71}$. The concert of multiscale fluid-solid interactions in brain tissue could 
make in vivo sSMRE at ultra-low drive frequencies especially attractive as a guidance for biomaterial engineering and as a clinical imaging marker of neurovascular diseases such as neurovascular dementia, cerebral pseudotumor, or hydrocephalus.

Although encouraging, our study is limited by one-dimensional analysis of large-scale wavelengths. The accepted method for suppression of compression waves is to apply a finite-difference curl operator despite well-known issues with noise amplification in the low-frequency range and discretization artifacts at lower spatial support72. For these reasons, we chose to analyze our data by wave fits which are unaffected by limitations of discretization models intrinsic to finite difference operators or finite element schemes 24 . Single-order finite difference operators as used in phase gradient-based inversions $\underline{73}$ have been proven relatively stable against noise and discretization $\underline{72}$. A 2D-extension of our analysis is presented in the supplementary material giving similar results of superviscous brain properties. Furthermore, shear waves in the brain are directional, heterogeneous, nonlinear, dispersive, and time-dependent due to the anisotropic, heterogeneous, nonlinear, and time-varying nature of the pulsating brain24 $\underline{30}$. No currently available technique can address all of these challenges at the same time. The proposed sSMRE techniques and large-scale analysis selectively addressed pulsation, dispersion, and viscoelastic model identification to provide the data that could guide future developments in 3D-MRE-based mapping of viscoelastic parameters of the brain in the ultra-low frequency range.

In summary, we have introduced ssMRE for quantifying SWS of in vivo human brain over a large frequency range from heart rate to an external driving frequency of $40 \mathrm{~Hz}$. The validity of measurement, postprocessing, and viscoelastic model fitting was demonstrated by numerical simulations and phantom data. Viscoelastic model fitting revealed brain tissue in the ultra-low frequency range to be dominated by superviscous properties with very low shear modulus of $42 \pm 13 \mathrm{~Pa}$ and relatively high viscosity of $6.6 \pm 0.3 \mathrm{~Pa} \cdot \mathrm{s}$. Our data explain why quasi-static measurement of brain mechanical parameters differed by orders of magnitude from in vivo MRE and shed light on the crucial role of fluid compartments including blood vessels and cerebrospinal fluid for whole brain properties. In vivo MRE at 
ultra-low frequencies has great potential as a diagnostic modality sensitive to changes associated with vascular neurological disorders and helps to model in vivo brain tissue biomaterial.

\section{Acknowledgments}

Funding from the German Research Foundation (GRK 2260 BIOQIC, SFB1340 Matrix in Vision) and from the European Union's Horizon 2020 Program (ID 668039, EU FORCE - Imaging the Force of Cancer) is gratefully acknowledged.

\section{Declaration of conflicting interests}

The authors declare no potential conflicts of interest with respect to the research, authorship, and publication of this article.

\section{Author contributions}

Helge Herthum as first author carried out all experiments and contributed to all parts of the manuscript. Sergio C H Dempsey and Abbas Samani carried out the FEM simulations. Felix Schrank and Mehrgan Shahryari contributed to the study design and experimental setup. Carsten Warmuth carried out the MRI sequence programming. Heiko Tzschätzsch helped to carry out the data analysis and verified the results. Jürgen Braun helped supervise the project and constructed the actuation system. Ingolf Sack designed and directed the project and aided in interpreting the results. All authors provided critical feedback and helped shape the research and manuscript.

\section{References}

1 Guo, J., Bertalan, G., Meierhofer, D., Klein, C., Schreyer, S., Steiner, B., Wang, S., Vieira da Silva, R., Infante-Duarte, C., Koch, S., Boehm-Sturm, P., Braun, J. \& Sack, I. Brain maturation is associated with increasing tissue stiffness and 
decreasing tissue fluidity. Acta Biomater 99, 433-442, doi:10.1016/j.actbio.2019.08.036 (2019).

2 Sack, I., Beierbach, B., Wuerfel, J., Klatt, D., Hamhaber, U., Papazoglou, S., Martus, P. \& Braun, J. The impact of aging and gender on brain viscoelasticity. Neuroimage 46, 652-657, doi:10.1016/j.neuroimage.2009.02.040 (2009).

3 Arani, A., Murphy, M. C., Glaser, K. J., Manduca, A., Lake, D. S., Kruse, S. A., Jack, C. R., Jr., Ehman, R. L. \& Huston, J., 3rd. Measuring the effects of aging and sex on regional brain stiffness with MR elastography in healthy older adults. Neuroimage 111, 59-64, doi:10.1016/j.neuroimage.2015.02.016 (2015).

4 Weickenmeier, J., de Rooij, R., Budday, S., Ovaert, T. C. \& Kuhl, E. The mechanical importance of myelination in the central nervous system. $J$ Mech Behav Biomed Mater 76, 119-124, doi:10.1016/j.jmbbm.2017.04.017 (2017).

5 Patz, S., Fovargue, D., Schregel, K., Nazari, N., Palotai, M., Barbone, P. E., Fabry, B., Hammers, A., Holm, S., Kozerke, S., Nordsletten, D. \& Sinkus, R. Imaging localized neuronal activity at fast time scales through biomechanics. Sci Adv 5, eaav3816, doi:10.1126/sciadv.aav3816 (2019).

6 Lan, P. S., Glaser, K. J., Ehman, R. L. \& Glover, G. H. Imaging brain function with simultaneous BOLD and viscoelasticity contrast: fMRI/fMRE. Neuroimage 211, 116592, doi:10.1016/j.neuroimage.2020.116592 (2020).

7 Schwarb, H., Johnson, C. L., Daugherty, A. M., Hillman, C. H., Kramer, A. F., Cohen, N. J. \& Barbey, A. K. Aerobic fitness, hippocampal viscoelasticity, and relational memory performance. Neuroimage 153, 179-188, doi:10.1016/j.neuroimage.2017.03.061 (2017).

8 Hiscox, L. V., Johnson, C. L., McGarry, M. D. J., Schwarb, H., van Beek, E. J. R., Roberts, N. \& Starr, J. M. Hippocampal viscoelasticity and episodic memory performance in healthy older adults examined with magnetic resonance elastography. Brain Imaging Behav 14, 175-185, doi:10.1007/s11682-018-9988-8 (2020).

9 Hetzer, S., Hirsch, S., Braun, J., Sack, I. \& Weygandt, M. Viscoelasticity of striatal brain areas reflects variations in body mass index of lean to overweight male 
adults. Brain Imaging Behav 14, 2477-2487, doi:10.1007/s11682-019-00200-w (2020).

10 Hetzer, S., Birr, P., Fehlner, A., Hirsch, S., Dittmann, F., Barnhill, E., Braun, J. \& Sack, I. Perfusion alters stiffness of deep gray matter. J Cereb Blood Flow Metab 38, 116-125, doi:10.1177/0271678X17691530 (2018).

11 Hetzer, S., Dittmann, F., Bormann, K., Hirsch, S., Lipp, A., Wang, D. J., Braun, J. \& Sack, I. Hypercapnia increases brain viscoelasticity. J Cereb Blood Flow Metab 39, 2445-2455, doi:10.1177/0271678X18799241 (2019).

12 Jamin, Y., Boult, J. K. R., Li, J., Popov, S., Garteiser, P., Ulloa, J. L., Cummings, C., Box, G., Eccles, S. A., Jones, C., Waterton, J. C., Bamber, J. C., Sinkus, R. \& Robinson, S. P. Exploring the biomechanical properties of brain malignancies and their pathologic determinants in vivo with magnetic resonance elastography. Cancer Res 75, 1216-1224, doi:10.1158/0008-5472.CAN-14-1997 (2015).

13 Streitberger, K. J., Lilaj, L., Schrank, F., Braun, J., Hoffmann, K. T., ReissZimmermann, M., Kas, J. A. \& Sack, I. How tissue fluidity influences brain tumor progression. Proc Natl Acad Sci $U$ S A 117, 128-134, doi:10.1073/pnas.1913511116 (2020).

14 Streitberger, K. J., Reiss-Zimmermann, M., Freimann, F. B., Bayerl, S., Guo, J., Arlt, F., Wuerfel, J., Braun, J., Hoffmann, K. T. \& Sack, I. High-resolution mechanical imaging of glioblastoma by multifrequency magnetic resonance elastography. PLoS One 9, e110588, doi:10.1371/journal.pone.0110588 (2014).

15 Hughes, J. D., Fattahi, N., Van Gompel, J., Arani, A., Meyer, F., Lanzino, G., Link, M. J., Ehman, R. \& Huston, J. Higher-Resolution Magnetic Resonance Elastography in Meningiomas to Determine Intratumoral Consistency. Neurosurgery 77, 653-658; discussion 658-659, doi:10.1227/NEU.0000000000000892 (2015).

16 Yin, Z., Glaser, K. J., Manduca, A., Van Gompel, J. J., Link, M. J., Hughes, J. D., Romano, A., Ehman, R. L. \& Huston, J., 3rd. Slip Interface Imaging Predicts Tumor-Brain Adhesion in Vestibular Schwannomas. Radiology 277, 507-517, doi:10.1148/radiol.2015151075 (2015). 
17 Fehlner, A., Behrens, J. R., Streitberger, K. J., Papazoglou, S., Braun, J., Bellmann-Strobl, J., Ruprecht, K., Paul, F., Wurfel, J. \& Sack, I. Higher-resolution MR elastography reveals early mechanical signatures of neuroinflammation in patients with clinically isolated syndrome. J Magn Reson Imaging 44, 51-58, doi:10.1002/jmri.25129 (2016).

18 Wuerfel, J., Paul, F., Beierbach, B., Hamhaber, U., Klatt, D., Papazoglou, S., Zipp, F., Martus, P., Braun, J. \& Sack, I. MR-elastography reveals degradation of tissue integrity in multiple sclerosis. Neuroimage 49, 2520-2525, doi:10.1016/j.neuroimage.2009.06.018 (2010).

19 Streitberger, K. J., Sack, I., Krefting, D., Pfuller, C., Braun, J., Paul, F. \& Wuerfel, J. Brain viscoelasticity alteration in chronic-progressive multiple sclerosis. PLoS One 7, e29888, doi:10.1371/journal.pone.0029888 (2012).

20 Murphy, M. C., Huston, J., 3rd \& Ehman, R. L. MR elastography of the brain and its application in neurological diseases. Neuroimage 187, 176-183, doi:10.1016/j.neuroimage.2017.10.008 (2019).

21 Huston, J., 3rd, Murphy, M. C., Boeve, B. F., Fattahi, N., Arani, A., Glaser, K. J., Manduca, A., Jones, D. T. \& Ehman, R. L. Magnetic resonance elastography of frontotemporal dementia. J Magn Reson Imaging 43, 474-478, doi:10.1002/jmri.24977 (2016).

22 Murphy, M. C., Jones, D. T., Jack, C. R., Jr., Glaser, K. J., Senjem, M. L., Manduca, A., Felmlee, J. P., Carter, R. E., Ehman, R. L. \& Huston, J., 3rd. Regional brain stiffness changes across the Alzheimer's disease spectrum. Neuroimage Clin 10, 283-290, doi:10.1016/j.nicl.2015.12.007 (2016).

23 Gerischer, L. M., Fehlner, A., Kobe, T., Prehn, K., Antonenko, D., Grittner, U., Braun, J., Sack, I. \& Floel, A. Combining viscoelasticity, diffusivity and volume of the hippocampus for the diagnosis of Alzheimer's disease based on magnetic resonance imaging. Neuroimage Clin 18, 485-493, doi:10.1016/j.nicl.2017.12.023 (2018).

24 Hirsch, S., Braun, J. \& Sack, I. Magnetic Resonance Elastography: Physical Background And Medical Applications. (Wiley-VCH, 2017). 
25 Chatelin, S., Constantinesco, A. \& Willinger, R. Fifty years of brain tissue mechanical testing: from in vitro to in vivo investigations. Biorheology 47, 255-276, doi:10.3233/BIR-2010-0576 (2010).

26 Bilston, L. E., Liu, Z. \& Phan-Thien, N. Linear viscoelastic properties of bovine brain tissue in shear. Biorheology 34, 377-385, doi:10.1016/s0006355x(98)00022-5 (1997).

27 Hrapko, M., van Dommelen, J. A., Peters, G. W. \& Wismans, J. S. Characterisation of the mechanical behaviour of brain tissue in compression and shear. Biorheology 45, 663-676 (2008).

28 Bilston, L. E., Liu, Z. \& Phan-Thien, N. Large strain behaviour of brain tissue in shear: some experimental data and differential constitutive model. Biorheology $\mathbf{3 8}$, 335-345 (2001).

29 McGarry, M. D., Johnson, C. L., Sutton, B. P., Georgiadis, J. G., Van Houten, E. E., Pattison, A. J., Weaver, J. B. \& Paulsen, K. D. Suitability of poroelastic and viscoelastic mechanical models for high and low frequency MR elastography. Med Phys 42, 947-957, doi:10.1118/1.4905048 (2015).

30 McGarry, M. D. J., Van Houten, E., Guertler, C., Okamoto, R. J., Smith, D. R., Sowinski, D. R., Johnson, C. L., Bayly, P., Weaver, J. \& Paulsen, K. D. A heterogenous, time harmonic, nearly incompressible transverse isotropic finite element brain simulation platform for MR elastography. Phys Med Biol, doi:10.1088/1361-6560/ab9a84 (2020).

31 Solamen, L. M., McGarry, M. D., Tan, L., Weaver, J. B. \& Paulsen, K. D. Phantom evaluations of nonlinear inversion MR elastography. Phys Med Biol 63, 145021, doi:10.1088/1361-6560/aacb08 (2018).

32 Budday, S., Nay, R., de Rooij, R., Steinmann, P., Wyrobek, T., Ovaert, T. C. \& Kuhl, E. Mechanical properties of gray and white matter brain tissue by indentation. J Mech Behav Biomed Mater 46, 318-330, doi:10.1016/j.jmbbm.2015.02.024 (2015).

33 Nicolle, S., Lounis, M., Willinger, R. \& Palierne, J. F. Shear linear behavior of brain tissue over a large frequency range. Biorheology 42, 209-223 (2005). 
34 Christ, A. F., Franze, K., Gautier, H., Moshayedi, P., Fawcett, J., Franklin, R. J., Karadottir, R. T. \& Guck, J. Mechanical difference between white and gray matter in the rat cerebellum measured by scanning force microscopy. J Biomech 43, 2986-2992, doi:10.1016/j.jbiomech.2010.07.002 (2010).

35 Prange, M. T. \& Margulies, S. S. Regional, directional, and age-dependent properties of the brain undergoing large deformation. J Biomech Eng 124, 244252, doi:10.1115/1.1449907 (2002).

36 Bertalan, G., Guo, J., Tzschatzsch, H., Klein, C., Barnhill, E., Sack, I. \& Braun, J. Fast tomoelastography of the mouse brain by multifrequency single-shot MR elastography. Magn Reson Med 81, 2676-2687, doi:10.1002/mrm.27586 (2019).

37 Dittmann, F., Hirsch, S., Tzschatzsch, H., Guo, J., Braun, J. \& Sack, I. In vivo wideband multifrequency MR elastography of the human brain and liver. Magn Reson Med 76, 1116-1126, doi:10.1002/mrm.26006 (2016).

38 Garo, A., Hrapko, M., van Dommelen, J. A. \& Peters, G. W. Towards a reliable characterisation of the mechanical behaviour of brain tissue: The effects of postmortem time and sample preparation. Biorheology 44, 51-58 (2007).

39 Kaster, T., Sack, I. \& Samani, A. Measurement of the hyperelastic properties of ex vivo brain tissue slices. J Biomech 44, 1158-1163, doi:10.1016/j.jbiomech.2011.01.019 (2011).

40 Donnelly, B. in Proceedings of the 26th International Workshop on Biomechanics Research.

41 Budday, S., Sommer, G., Birkl, C., Langkammer, C., Haybaeck, J., Kohnert, J., Bauer, M., Paulsen, F., Steinmann, P., Kuhl, E. \& Holzapfel, G. A. Mechanical characterization of human brain tissue. Acta Biomater 48, 319-340, doi:10.1016/j.actbio.2016.10.036 (2017).

42 Prevost, T. P., Jin, G., de Moya, M. A., Alam, H. B., Suresh, S. \& Socrate, S. Dynamic mechanical response of brain tissue in indentation in vivo, in situ and in vitro. Acta Biomater 7, 4090-4101, doi:10.1016/j.actbio.2011.06.032 (2011).

43 Hiscox, L. V., Johnson, C. L., Barnhill, E., McGarry, M. D., Huston, J., van Beek, E. J., Starr, J. M. \& Roberts, N. Magnetic resonance elastography (MRE) of the 
human brain: technique, findings and clinical applications. Phys Med Biol 61, R401-R437, doi:10.1088/0031-9155/61/24/R401 (2016).

44 Testu, J., McGarry, M. D. J., Dittmann, F., Weaver, J. B., Paulsen, K. D., Sack, I. \& Van Houten, E. E. W. Viscoelastic power law parameters of in vivo human brain estimated by MR elastography. J Mech Behav Biomed Mater 74, 333-341, doi:10.1016/j.jmbbm.2017.06.027 (2017).

45 Weaver, J. B., Pattison, A. J., McGarry, M. D., Perreard, I. M., Swienckowski, J. G., Eskey, C. J., Lollis, S. S. \& Paulsen, K. D. Brain mechanical property measurement using MRE with intrinsic activation. Phys Med Biol 57, 7275-7287, doi:10.1088/0031-9155/57/22/7275 (2012).

46 Gordon-Wylie, S. W., Solamen, L. M., McGarry, M. D. J., Zeng, W., VanHouten, E., Gilbert, G., Weaver, J. B. \& Paulsen, K. D. MR elastography at $1 \mathrm{~Hz}$ of gelatin phantoms using 3D or 4D acquisition. J Magn Reson 296, 112-120, doi:10.1016/j.jmr.2018.08.012 (2018).

47 Schrank, F., Warmuth, C., Tzschatzsch, H., Kreft, B., Hirsch, S., Braun, J., Elgeti, T. \& Sack, I. Cardiac-gated steady-state multifrequency magnetic resonance elastography of the brain: Effect of cerebral arterial pulsation on brain viscoelasticity. J Cereb Blood Flow Metab 40, 991-1001, doi:10.1177/0271678X19850936 (2020).

48 Yamada, K. M. \& Cukierman, E. Modeling tissue morphogenesis and cancer in 3D. Cell 130, 601-610, doi:10.1016/j.cell.2007.08.006 (2007).

49 Blehm, B. H., Devine, A., Staunton, J. R. \& Tanner, K. In vivo tissue has non-linear rheological behavior distinct from 3D biomimetic hydrogels, as determined by AMOTIV microscopy. Biomaterials 83, 66-78, doi:10.1016/j.biomaterials.2015.12.019 (2016).

50 Ananthanarayanan, B., Kim, Y. \& Kumar, S. Elucidating the mechanobiology of malignant brain tumors using a brain matrix-mimetic hyaluronic acid hydrogel platform. Biomaterials 32, 7913-7923, doi:10.1016/j.biomaterials.2011.07.005 (2011). 
51 Ozawa, H., Matsumoto, T., Ohashi, T., Sato, M. \& Kokubun, S. Mechanical properties and function of the spinal pia mater. J Neurosurg Spine 1, 122-127, doi:10.3171/spi.2004.1.1.0122 (2004).

52 Reina, M. A., De Leon Casasola Ode, L., Villanueva, M. C., Lopez, A., Maches, F. \& De Andres, J. A. Ultrastructural findings in human spinal pia mater in relation to subarachnoid anesthesia. Anesth Analg 98, 1479-1485, table of contents, doi:10.1213/01.ane.0000113240.09354.e9 (2004).

53 Jin, X., Lee, J. B., Leung, L. Y., Zhang, L., Yang, K. H. \& King, A. I. Biomechanical response of the bovine pia-arachnoid complex to tensile loading at varying strainrates. Stapp Car Crash J 50, 637-649 (2006).

54 Barber, T. W., Brockway, J. A. \& Higgins, L. S. The density of tissues in and about the head. Acta Neurol Scand 46, 85-92, doi:10.1111/j.1600-0404.1970.tb05606.x (1970).

55 Dittmann, F., Reiter, R., Guo, J., Haas, M., Asbach, P., Fischer, T., Braun, J. \& Sack, I. Tomoelastography of the prostate using multifrequency MR elastography and externally placed pressurized-air drivers. Magn Reson Med 79, 1325-1333, doi:10.1002/mrm.26769 (2018).

56 Fehlner, A., Hirsch, S., Weygandt, M., Christophel, T., Barnhill, E., Kadobianskyi, M., Braun, J., Bernarding, J., Lutzkendorf, R., Sack, I. \& Hetzer, S. Increasing the spatial resolution and sensitivity of magnetic resonance elastography by correcting for subject motion and susceptibility-induced image distortions. J Magn Reson Imaging 46, 134-141, doi:10.1002/jmri.25516 (2017).

57 Schrank, F., Warmuth, C., Gorner, S., Meyer, T., Tzschatzsch, H., Guo, J., Uca, Y. O., Elgeti, T., Braun, J. \& Sack, I. Real-time MR elastography for viscoelasticity quantification in skeletal muscle during dynamic exercises. Magn Reson Med 84, 103-114, doi:10.1002/mrm.28095 (2020).

58 Aster, R. C., Borchers, B. \& Thurber, C. H. Parameter Estimation and Inverse Problems. (Elsevier, 2013).

59 Bland, J. M. \& Altman, D. G. Applying the right statistics: analyses of measurement studies. Ultrasound Obstet Gynecol 22, 85-93, doi:10.1002/uog.122 (2003). 
60 Sauer, F., Oswald, L., Ariza de Schellenberger, A., Tzschatzsch, H., Schrank, F., Fischer, T., Braun, J., Mierke, C. T., Valiullin, R., Sack, I. \& Kas, J. A. Collagen networks determine viscoelastic properties of connective tissues yet do not hinder diffusion of the aqueous solvent. Soft Matter 15, 3055-3064, doi:10.1039/c8sm02264j (2019).

61 Hirsch, S., Klatt, D., Freimann, F., Scheel, M., Braun, J. \& Sack, I. In vivo measurement of volumetric strain in the human brain induced by arterial pulsation and harmonic waves. Magn Reson Med 70, 671-683, doi:10.1002/mrm.24499 (2013).

62 Barnhill, E., Hollis, L., Sack, I., Braun, J., Hoskins, P. R., Pankaj, P., Brown, C., van Beek, E. J. R. \& Roberts, N. Nonlinear multiscale regularisation in MR elastography: Towards fine feature mapping. Med Image Anal 35, 133-145, doi:10.1016/j.media.2016.05.012 (2017).

63 Solamen, L. M., Gordon-Wylie, S. W., McGarry, M. D., Weaver, J. B. \& Paulsen, K. D. Phantom evaluations of low frequency MR elastography. Phys Med Biol 64, 065010, doi:10.1088/1361-6560/ab0290 (2019).

64 Tzschatzsch, H., Ipek-Ugay, S., Guo, J., Streitberger, K. J., Gentz, E., Fischer, T., Klaua, R., Schultz, M., Braun, J. \& Sack, I. In vivo time-harmonic multifrequency elastography of the human liver. Phys Med Bio/59, 1641-1654, doi:10.1088/00319155/59/7/1641 (2014).

65 Bouvy, W. H., Geurts, L. J., Kuijf, H. J., Luijten, P. R., Kappelle, L. J., Biessels, G. J. \& Zwanenburg, J. J. Assessment of blood flow velocity and pulsatility in cerebral perforating arteries with 7-T quantitative flow MRI. NMR Biomed 29, 1295-1304, doi:10.1002/nbm.3306 (2016).

66 Rashid, S., McAllister, J. P., 2nd, Yu, Y. \& Wagshul, M. E. Neocortical capillary flow pulsatility is not elevated in experimental communicating hydrocephalus. $J$ Cereb Blood Flow Metab 32, 318-329, doi:10.1038/jcbfm.2011.130 (2012).

67 Tully, B. \& Ventikos, Y. Coupling poroelasticity and CFD for cerebrospinal fluid hydrodynamics. IEEE Trans Biomed Eng 56, 1644-1651, doi:10.1109/TBME.2009.2016427 (2009). 
68 Parker, K. J. Are rapid changes in brain elasticity possible? Phys Med Biol 62, 7425-7439, doi:10.1088/1361-6560/aa8380 (2017).

69 Weickenmeier, J., Kurt, M., Ozkaya, E., de Rooij, R., Ovaert, T. C., Ehman, R. L., Butts Pauly, K. \& Kuhl, E. Brain stiffens post mortem. Journal of the Mechanical Behavior of Biomedical Materials 84, 88-98, doi:https://doi.org/10.1016/j.jmbbm.2018.04.009 (2018).

70 Bertalan, G., Klein, C., Schreyer, S., Steiner, B., Kreft, B., Tzschatzsch, H., de Schellenberger, A. A., Nieminen-Kelha, M., Braun, J., Guo, J. \& Sack, I. Biomechanical properties of the hypoxic and dying brain quantified by magnetic resonance elastography. Acta Biomater 101, 395-402, doi:10.1016/j.actbio.2019.11.011 (2020).

71 Parker, K. J. A microchannel flow model for soft tissue elasticity. Phys Med Biol 59, 4443-4457, doi:10.1088/0031-9155/59/15/4443 (2014).

72 Mura, J., Schrank, F. \& Sack, I. An analytical solution to the dispersion-byinversion problem in magnetic resonance elastography. Magnetic Resonance in Medicine 84, 61-71, doi:10.1002/mrm.28247 (2020).

73 Tzschatzsch, H., Guo, J., Dittmann, F., Hirsch, S., Barnhill, E., Johrens, K., Braun, J. \& Sack, I. Tomoelastography by multifrequency wave number recovery from time-harmonic propagating shear waves. Med Image Anal 30, 1-10, doi:10.1016/j.media.2016.01.001 (2016). 


\section{Supplementary material to}

\section{Superviscous properties of the in vivo brain at large scales.}

Helge Herthum ${ }^{1}$, Sergio C H Dempsey², Abbas Samani ${ }^{2,3,4}$, Felix Schrank ${ }^{1}$, Mehrgan Shahryari ${ }^{1}$, Carsten Warmuth ${ }^{1}$, Jürgen Braun ${ }^{5}$, Heiko Tzschätzsch ${ }^{1}$, Ingolf Sack ${ }^{1, *}$

1'Department of Radiology, Charité - Universitätsmedizin Berlin, Berlin, Germany ${ }^{2}$ School of Biomedical Engineering, Western University, London, Ontario, Canada

${ }^{3}$ Department of Medical Biophysics, Western University, London, Ontario, Canada

${ }^{4}$ Department of Electrical and Computer Engineering, Western University, London, Ontario, Canada

${ }^{5}$ Institute of Medical Informatics, Charité - Universitätsmedizin Berlin, Berlin, Germany

\section{${ }^{*}$ Corresponding author:}

Ingolf Sack, $\mathrm{PhD}$

Department of Radiology

Charité - Universitätsmedizin Berlin

Charitéplatz 1

10117 Berlin, Germany

Tel +4930450539058

Ingolf.sack@charite.de

We here present a complementary data analysis that uses a $2 \mathrm{D}$ mapping inversion technique to arrive at values similar to those described in the main manuscript. We consider 1D-wave profile fitting as a ground-truth method for the reasons described in the main manuscript. Nevertheless, the possibility to obtain meaningful parameter maps without manual profile selection would facilitate low-frequency MRE in future clinical applications. 


\section{Data analysis by 2D SWS inversion}

As described in the main manuscript, ground truth shear wave speed (SWS) was determined by profile fitting and reproduced by SWS mapping based on frequencyadaptive multi-component wave-number inversion $\underline{1}$. The denoising parameters prior to inversion were calibrated in such a way that the results would reproduce estimates of SWS in the heparin phantom shown in Figure 5 in the main document. Averaged SWS values over frequency were added to the previously shown phantom data and plotted in supplementary Figure 1. The procedure of SWS mapping at low frequencies and the obtained results are presented in the following chapters.

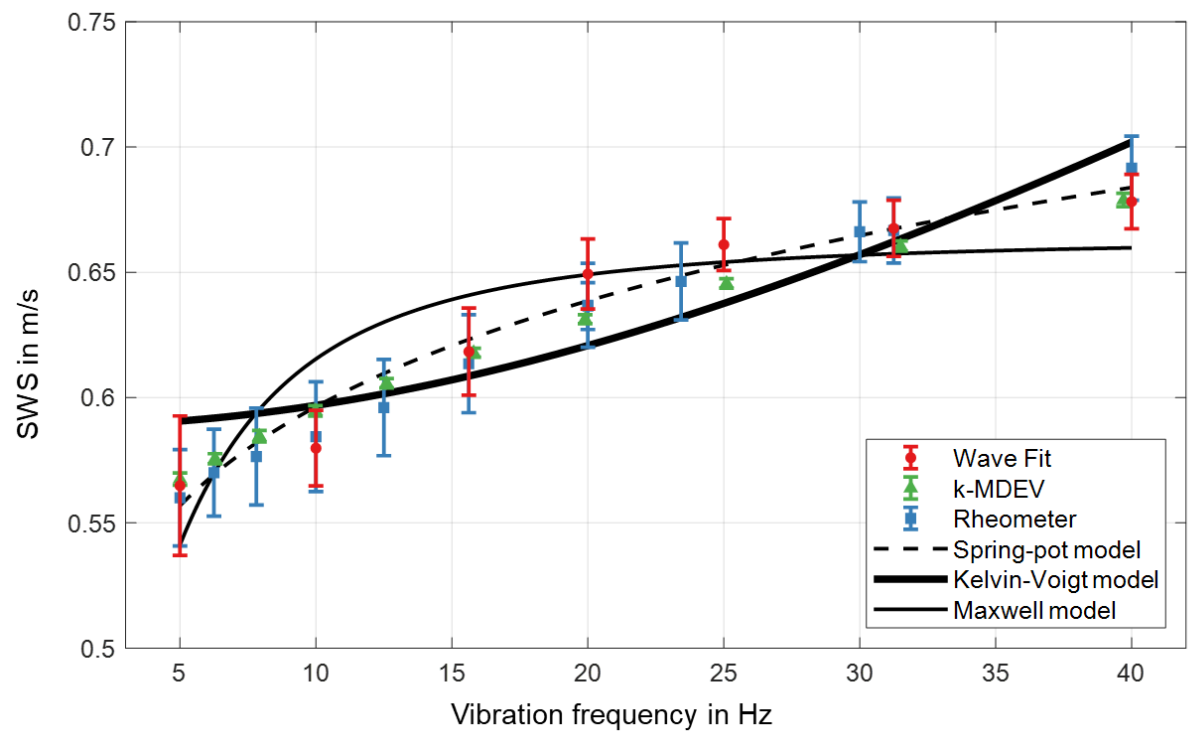

Supplementary Figure 1: SWS in heparin gel phantom for different vibration frequencies $(5-40 \mathrm{~Hz})$. SWS was quantified by sSMRE and 1D profile-based fits using bidirectional plane waves as model function, as well as mapping by $k$-MDEV inversion. Ground truth values were determined by shear oscillation rheometry. In addition, different rheological models were fitted to the profile-based SSMRE data.

\section{Methods}

For automatic data processing and parameter reconstruction single-gradient wavenumber recovery was applied to avoid noise amplification of the Laplacian which is invoked by direct inversion techniquesz' $\underline{3}$. The principle of wavenumber $(k-)$ based multicomponent, elasto-visco ( $k$-MDEV) inversion is outlined in $\underline{1}$. $k$-MDEV generates maps of 
SWS, which is related to tissue stiffness. This method was adapted to the brain by the following steps:

(i) Similar to $\underline{1}$, the complex MRI signal was smoothed before phase unwrapping; however, instead of Gaussian denoising, a low-pass Butterworth filter of order 3 with an upper threshold of $230 / \mathrm{m}$ was applied.

(ii) 2D Laplacian-based phase unwrapping as described in $\underline{4}$ was used and combined with temporal Fourier transformation to extract the complex-valued wave field of a single harmonic frequency. For intrinsic activation sSMRE, the frequency bin corresponding to the heart rate of the volunteer (approximately $1 \mathrm{~Hz}$ ) was selected.

(iii) Since unavoidable for SWS mapping, directional filtering was applied as explained in 1. However, here we replaced the high-pass filter with a Butterworth filter of order 3 and adaptive threshold values empirically determined by phantom experiments according to the excitation frequency in order to eliminate unwanted signal at low wave numbers. To this end, a function of the lower filter bound was derived to compute the lower threshold of the high-pass Butterworth filter for a given frequency in the range between 6.25 and 20 $\mathrm{Hz}$ (supplementary Figure 2). For frequencies below or above that range, threshold values were used corresponding to $6.25 \mathrm{~Hz}$ or $20 \mathrm{~Hz}$.

SWS was quantified by averaging values over regions of interest (ROls) which were manually drawn for whole brain parenchyma excluding ventricles using anatomical T2weighted MRE magnitude images as depicted in the image slice.

Nine healthy volunteers were investigated a second time to assess the repeatability of in vivo ssMRE at $5 \mathrm{~Hz}$ and $10 \mathrm{~Hz}$ vibration frequency. This was analyzed by intraclass correlation coefficient (ICC) accounting for two-way mixed effects.

$$
I C C=\frac{M S_{S}-M S_{E}}{M S_{S}+M S_{E}}
$$

$M S_{S}$ denotes the mean squares between subjects and $M S_{E}$ is the residual mean squares. 


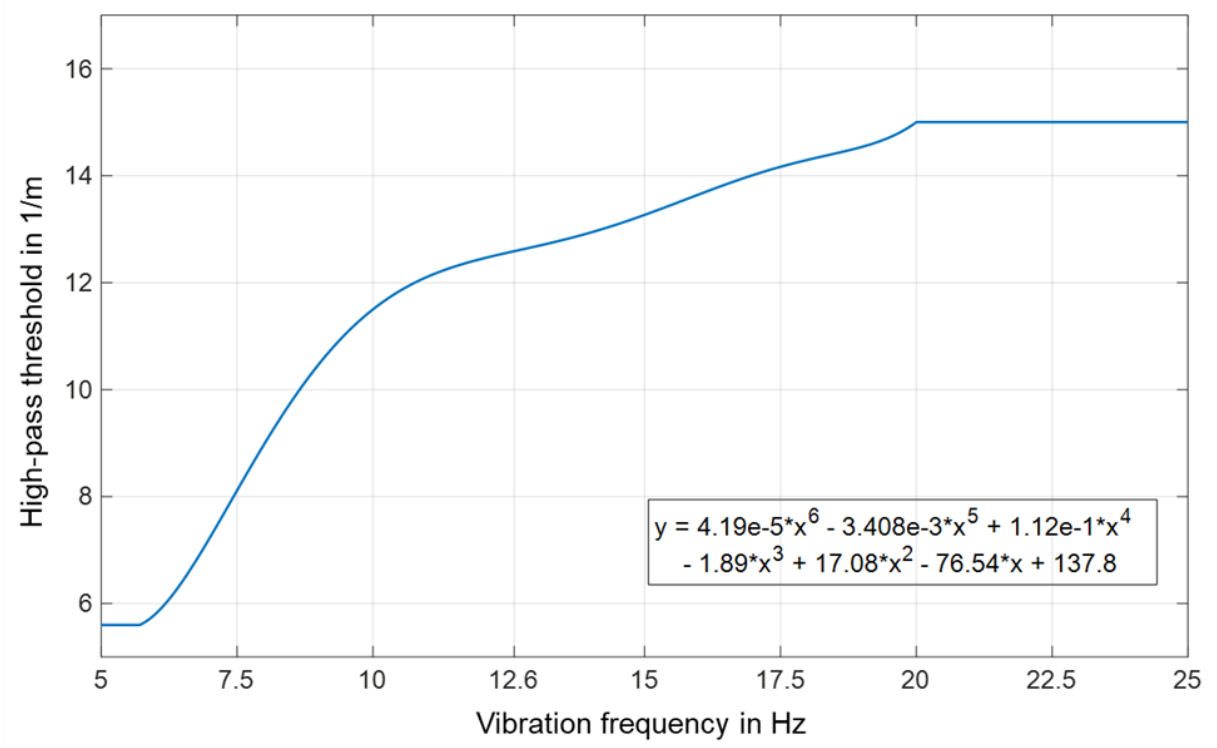

Supplementary Figure 2: Continuous threshold function for the low-pass Butterworth filter threshold versus frequency. Thresholds for frequencies below $6.25 \mathrm{~Hz}$ or above $20 \mathrm{~Hz}$ are identical to those of these respective frequencies.

\section{Results}

Supplementary Figure 3 shows the series of SWS maps of SSMRE for one volunteer. The strong dispersion of SWS values from 5 to $40 \mathrm{~Hz}$ is indicated by the increasing intensity in SWS maps and larger ranges of the grayscale bars at higher frequencies. Lower frequencies suffer from pronounced noise in the elastogram.

Averaged SWS values over frequency are added to the previously shown data and plotted in supplementary Figure 4. Both, profile-based SWS recovery and $k$-MDEV inversion fall in the same range of values with similar dispersion functions over frequency. Dispersion curve of SWS in the human brain obtained using a profile-based fitting routine and $k$ MDEV inversion for every frequency including intrinsic activation ssMRE. For comparison, values reported by Dittmann et al. $\underline{\underline{4}}$ and Testu et al. $\underline{5}$ are shown. Two rheological models, the viscous model and the Kelvin-Voigt model (see Table 1), were fitted to the SWS data obtained by $1 \mathrm{D}$ profile fitting. 

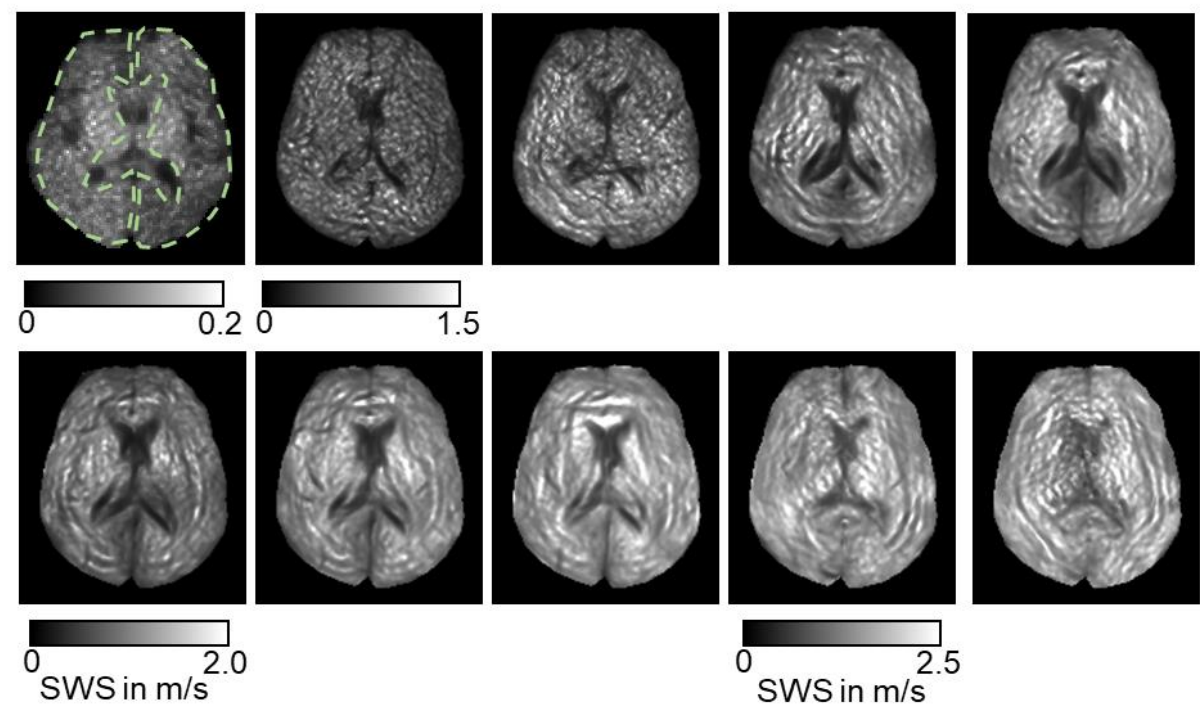

Supplementary Figure 3: SWS maps generated by 2D k-MDEV inversion. Order from top left: Intrinsically activated MRE, $5 \mathrm{~Hz}, 6.25 \mathrm{~Hz}, 7.8125 \mathrm{~Hz}, 10 \mathrm{~Hz}, 12.5 \mathrm{~Hz}, 15.625 \mathrm{~Hz}, 20 \mathrm{~Hz}, 30 \mathrm{~Hz}, 40 \mathrm{~Hz}$. A ROI covering the brain parenchyma without ventricles for spatial averaging values is shown in the first image.

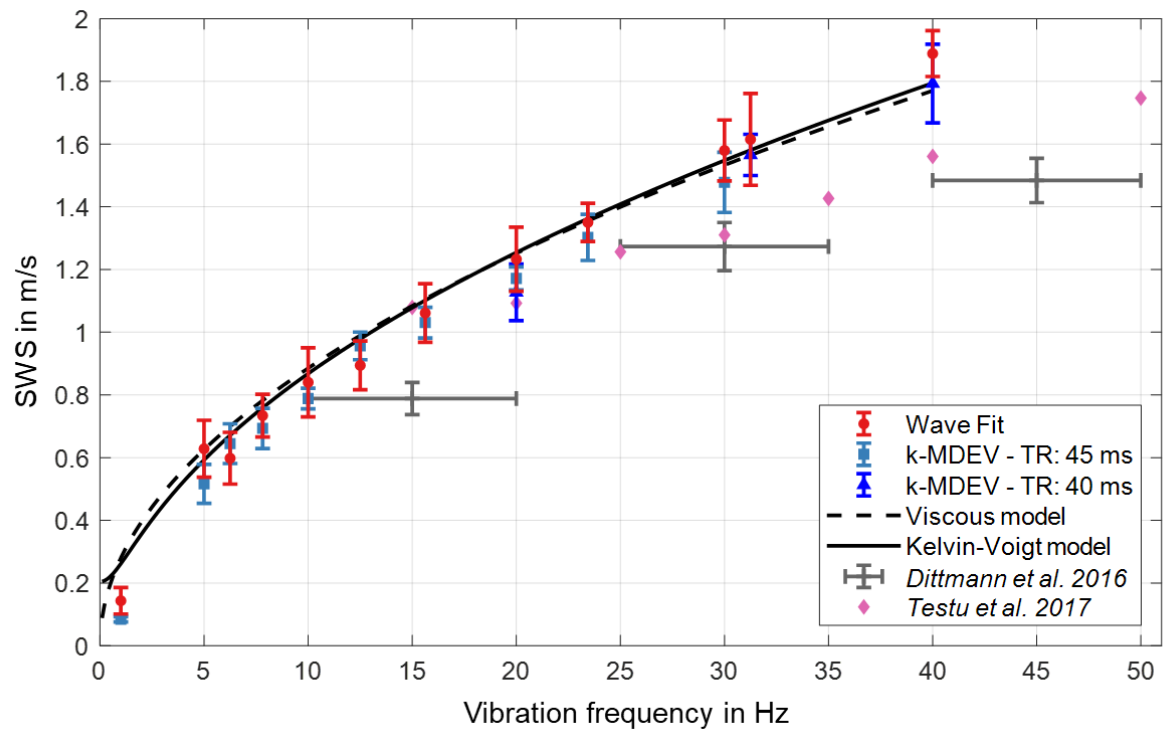

Supplementary Figure 4: Dispersion curve of SWS in the human brain obtained using a profile-based fitting routine and $k$-MDEV inversion for every frequency including intrinsic activation sSMRE. For comparison, values reported by Dittmann et al. $\underline{4}$ and Testu et al. $\underline{5}$ are shown. Two rheological models, the viscous model and the Kelvin-Voigt model (see Table 1 ), were fitted to the SWS data obtained by 1D profile fitting. 
Group mean SWS values of $k$-MDEV are mostly lower than those of slope fitting probably because of underestimation of values by derivative gradients invoked by the $k$-MDEV inversion $\underline{3}$. Nevertheless, the order of values in the range of $0.1 \mathrm{~m} / \mathrm{s}$ consistently indicates the very soft brain properties in that ultra-low frequency range.

The ICC for reproducibility of SsMRE gives values of 0.86 at $5 \mathrm{~Hz}$ and 0.71 at $10 \mathrm{~Hz}$. For computing the ICC, measurements had to be repeated twice. Our ethics committee advised us to focus with test-retest examinations on two novel biomarkers, which we chose with 5 and $10 \mathrm{~Hz}$.

\section{Discussion}

The ICC suggests at least good reproducibility of automatic 2D SWS inversion of ssMRE values for 5 and $10 \mathrm{~Hz}$, which are both outside the range that has been explored in vivo so far. Previous work on in vivo wideband MRE of the human brain has demonstrated the technical feasibility of using $10 \mathrm{~Hz}$ as drive frequency in MRE while being compromised in that specific frequency range $\underline{4} \underline{5}$. One possible factor contributing to the failure of other inversion techniques for brain MRE at ultra-low wave numbers might be the small wave curvature relative to noisez. Therefore, we used profile-based fits as ground truth for the adaptation of our $k$-MDEV inversion. $k$-MDEV shows good agreement in both the phantom studies and the in vivo brain examinations, suggesting that inversion techniques are feasible in ultra-low frequency MRE. Direct inversion techniques invoke second-order derivative gradients, which are more sensitive to noise and underestimate values in the range of low wave numbers as compared with $k-M D E V \underline{6}$. This is probably the reason why we obtained smaller values by direct inversion in $\underline{4}$ than in our present study, while data obtained by nonlinear inversions showed good agreement with $k$-MDEV. Nevertheless, this preliminary analysis shows the feasibility of noise-robust wave inversion in wideband brain MRE when tailored to a wider frequency range from $1 \mathrm{~Hz}$ to $40 \mathrm{~Hz}$.

The results we obtained with conventional drive frequencies of $20 \mathrm{to} 40 \mathrm{~Hz}$ are within the range of published results $\underline{8}$. However, we note a discrepancy with results published for intrinsic activation MRE of the braing, which are on the order of $2.4 \mathrm{kPa}$. The higher values 
might reflect the underlying poroelastic interactions modeled ing, while we accounted for the effective-medium shear modulus. The speed of the propagating shear waves emanating from larger arteries in the circle of Willis are visualized by the animated images accompanying this document as well as in the $\mathrm{x}$-t plot shown in Figure $6 \mathrm{C}$ of the main document. The slope of the propagating wave clearly indicates a wave speed of approximately $0.1 \mathrm{~m} / \mathrm{s}$, corresponding to a very low shear modulus of only 42Pa when the elastic model is used.

\section{References}

1 Tzschatzsch, H., Guo, J., Dittmann, F., Hirsch, S., Barnhill, E., Johrens, K., Braun, J. \& Sack, I. Tomoelastography by multifrequency wave number recovery from time-harmonic propagating shear waves. Med Image Anal 30, 1-10, doi:10.1016/j.media.2016.01.001 (2016).

2 Hirsch, S., Braun, J. \& Sack, I. Magnetic Resonance Elastography: Physical Background And Medical Applications. (Wiley-VCH, 2017).

3 Mura, J., Schrank, F. \& Sack, I. An analytical solution to the dispersion-byinversion problem in magnetic resonance elastography. Magn Reson Med 84, 6171, doi:10.1002/mrm.28247 (2020).

4 Dittmann, F., Hirsch, S., Tzschatzsch, H., Guo, J., Braun, J. \& Sack, I. In vivo wideband multifrequency MR elastography of the human brain and liver. Magn Reson Med 76, 1116-1126, doi:10.1002/mrm.26006 (2016).

5 Testu, J., McGarry, M. D. J., Dittmann, F., Weaver, J. B., Paulsen, K. D., Sack, I. \& Van Houten, E. E. W. Viscoelastic power law parameters of in vivo human brain estimated by MR elastography. J Mech Behav Biomed Mater 74, 333-341, doi:10.1016/j.jmbbm.2017.06.027 (2017).

6 Bertalan, G., Guo, J., Tzschatzsch, H., Klein, C., Barnhill, E., Sack, I. \& Braun, J. Fast tomoelastography of the mouse brain by multifrequency single-shot MR elastography. Magn Reson Med 81, 2676-2687, doi:10.1002/mrm.27586 (2019).

7 Barnhill, E., Hollis, L., Sack, I., Braun, J., Hoskins, P. R., Pankaj, P., Brown, C., van Beek, E. J. R. \& Roberts, N. Nonlinear multiscale regularisation in MR 
elastography: Towards fine feature mapping. Med Image Anal 35, 133-145, doi:10.1016/j.media.2016.05.012 (2017).

8 Hiscox, L. V., Johnson, C. L., Barnhill, E., McGarry, M. D., Huston, J., van Beek, E. J., Starr, J. M. \& Roberts, N. Magnetic resonance elastography (MRE) of the human brain: technique, findings and clinical applications. Phys Med Biol 61, R401-R437, doi:10.1088/0031-9155/61/24/R401 (2016).

9 Weaver, J. B., Pattison, A. J., McGarry, M. D., Perreard, I. M., Swienckowski, J. G., Eskey, C. J., Lollis, S. S. \& Paulsen, K. D. Brain mechanical property measurement using MRE with intrinsic activation. Phys Med Biol 57, 7275-7287, doi:10.1088/0031-9155/57/22/7275 (2012). 